\title{
On extreme-ultraviolet helium line intensity enhancement factors on the sun
}

\author{
A. S. Giunta, A. Fludra, A. C. Lanzafame, \\ M. G. O'Mullane, H. P. Summers, and W. Curdt
}

\section{Published version information}

Citation: Giunta, AS et al. "On extreme-ultraviolet helium line intensity enhancement factors on the sun." Astrophysical Journal, vol. 803, no. 2 (2015): 66

doi: http://dx.doi.org/10.1088/0004-637X/803/2/66

This version is made available in accordance with publisher policies. Please cite only the published version using the reference above. 


\title{
ON EXTREME-ULTRAVIOLET HELIUM LINE INTENSITY ENHANCEMENT FACTORS ON THE SUN
}

\author{
A. S. Giunta ${ }^{1}$, A. Fludra ${ }^{1}$, A. C. Lanzafame ${ }^{2}$, M. G. O’Mullane ${ }^{3}$, H. P. Summers ${ }^{3}$, and W. Curdt ${ }^{4}$ \\ ${ }^{1}$ STFC Rutherford Appleton Laboratory, Chilton, Didcot, Oxon. OX11 0QX, UK; alessandra.giunta@stfc.ac.uk \\ ${ }^{2}$ Dipartimento di Fisica e Astronomia, Università di Catania, via S. Sofia 78, I-95123 Catania, Italy \\ ${ }^{3}$ Department of Physics, University of Strathclyde, 107 Rottenrow, Glasgow G4 0NG, UK \\ ${ }^{4}$ Max-Planck-Institut für Sonnensystemforschung, Max-Planck-Str. 2, D-37191 Katlenburg-Lindau, Germany \\ Received 2014 June 27; accepted 2015 February 16; published 2015 April 17
}

\begin{abstract}
Helium lines in the solar EUV spectrum provide useful diagnostics of the solar atmosphere plasma. Helium is one of the few elements that exhibits strong emission lines formed in the lower transition region, and it is the second most abundant element in the Sun. However, the analysis of helium lines is complicated by their optical thickness and the unusual behavior of their intensities, with enhancements by a factor of up to 15 reported in the literature. Detailed study requires spatially and spectrally resolved observations in the EUV range, as well as sophisticated atomic modeling. The present work focuses on the application of the differential emission measure distribution to reproduce the observed fluxes of the $\mathrm{He}$ I and $\mathrm{He}$ II lines observed by the Solar and Heliospheric Observatory $(\mathrm{SOHO}) /$ Coronal Diagnostic Spectrometer and Hinode/EIS spectrometers, using the latest atomic data. It is found that the comparison between observed and reconstructed intensities for He I resonance lines $\lambda \lambda 537.03,522.21$, and 515.62 and the intercombination line $\lambda 591.41$ does not show a real enhancement. By contrast, He i $\lambda 584.33$, the first line of the $1 \mathrm{~s}^{2}{ }^{1} \mathrm{~S}-1 \mathrm{~s} n \mathrm{p}{ }^{1} \mathrm{P}$ resonance series, shows a depletion of a factor $\sim 2$, due to the opacity effect, as supported by non-LTE radiative transfer calculations. For single ionized helium lines 303.78 and $256.32 \AA$, the enhancement factors obtained are higher and agree with those of previous work. The different behavior of $\mathrm{He}$ I and $\mathrm{He}$ II lines suggests a mechanism that affects ionized helium only.
\end{abstract}

Key words: atomic data - Sun: atmosphere - Sun: chromosphere - Sun: transition region - Sun: UV radiation techniques: spectroscopic

\section{INTRODUCTION}

The early studies on helium formation mechanisms in the Sun date back to 1939 (Goldberg 1939), but the first quantitative demonstration of anomalous high intensities of the neutral and single ionized EUV helium lines, based on emission measure distribution, comes from Jordan (1975). She found that, under optically thin conditions, the reconstructed integrated emission of He I $584.33 \AA$ was a factor of 15 lower than the observed intensity, using disk-averaged solar spectra. Similarly, she derived an enhancement factor of 5.5 for He II 303.78 A. These results were updated by Macpherson \& Jordan (1999), using spatially resolved observations of quiet regions performed by SOHO (Solar and Heliospheric Observatory) instrumentations (Solar Measurement of Emitted Radiation, SUMER, and Coronal Diagnostic Spectrometer, CDS, to derive the emission measure distribution, combined with the EUV Imaging Telescope, EIT, and the Michelson Doppler Imager), which allowed the analysis of supergranulation boundaries and cell interior structures. Furthermore, the atomic data of Arnaud \& Rothenflug (1985) for He II and from CHIANTI v.1 (Dere et al. 1997) were adopted in their work, whereas Jordan (1975) used Athay (1960) and Hearn (1969) calculations for the helium atomic population. Macpherson \& Jordan (1999) found that $\mathrm{He}_{\text {I }} 584.33$ and $\mathrm{He}$ II $303.78 \AA$ lines were enhanced by factors of 10 and 13, respectively, in the cell boundaries and by factors of 14 and 25 , respectively, in the cell interiors. Hence, while the enhancement factors of the $\mathrm{He}_{\text {I }} 584.33 \AA$ line were quite similar in the two studies, higher enhancements by a factor of 2-4 were derived for the He II $303.78 \AA$ line. More recently, helium enhancement factors have been revised by Pietarila \& Judge (2004). They noted that previous studies of helium enhancements recognized the need for a treatment of radiative transfer in the relatively opaque helium lines, but did not include the possible effects of opacity on the lines used in the underlying emission measure analysis. Therefore, they performed new calculations of the intensities of lines of helium, carbon, oxygen, and silicon, using two different approaches. The first approach was the optically thin approximation used by Jordan (1975), while in the second approach the non-LTE transfer equation was solved together with the statistical equilibrium equations. In order to perform their calculations, Pietarila \& Judge (2004) used atomic models of helium, carbon, oxygen, and silicon, taking energy levels, collisional and radiative data from CHIANTI (v. 3-4), and ionization data from Arnaud \& Rothenflug (1985) for ground-level ionization and from the general formula of Burgess \& Chidichimo (1983) for excited level ionization. Using optically thin calculations and making some adjustment to account for the behavior of other lines formed at similar temperatures as helium lines, they obtained enhancement factors of 3 and 13 for $\mathrm{He}_{\text {I }} 584.33$ and He II $303.78 \AA$ A, respectively. In the second type of calculations, one-dimensional atmospheric models specifying thermal properties as a function of column mass were built up and full nonLTE transfer solutions were made using the MULTI code (Carlsson 1986). The results obtained from the full radiative transfer showed that $\mathrm{He}_{\mathrm{I}} 584.33, \mathrm{He}_{\mathrm{I}} 537.03 \AA$, and $\mathrm{He}$ II $303.78 \AA$ were enhanced by factors of $2-5,4-7$, and $2-9$ respectively, depending on the elemental abundances assumed for heavier elements. The revisit of helium enhancements proposed by Pietarila \& Judge (2004) shows an improvement of the ratio between observed and predicted intensities compared with the previous work of about a factor of 3 for both $\mathrm{He} \mathrm{I}$ and $\mathrm{He}$ II. While it is difficult to explain a discrepancy of a factor of 10 or more between observed and predicted 
Table 1

Details of the Joint SUMER, CDS, EIS Observations

\begin{tabular}{lccc}
\hline \hline $\begin{array}{l}\text { Observing } \\
\text { Parameters }\end{array}$ & SUMER & CDS & EIS \\
\hline Start & $12: 00 \mathrm{UT}$ & $12: 00 \mathrm{UT}$ & $12: 02 \mathrm{UT}$ \\
End & $15: 17 \mathrm{UT}$ & $16: 37 \mathrm{UT}$ & $15: 52 \mathrm{UT}$ \\
Pointing & $\left(0^{\prime \prime},-40^{\prime \prime}\right)$ & $\left(-1^{\prime \prime},-40^{\prime \prime}\right)$ & $\left(0.0^{\prime \prime},-26^{\prime \prime}\right)$ \\
Slit & $1^{\prime \prime} \times 120^{\prime \prime}$ & $2^{\prime \prime} \times 240^{\prime \prime}$ & $1^{\prime \prime} \times 256^{\prime \prime}$ \\
FOV & $90^{\prime \prime} \times 120^{\prime \prime}$ & $80^{\prime \prime} \times 240^{\prime \prime}$ & $60^{\prime \prime} \times 256^{\prime \prime}$ \\
Exp. time & $30 \mathrm{~s}$ & $55 \mathrm{~s}$ & $40 \mathrm{~s}$ \\
Windows & 19 & 14 & 7 \\
Scans & 2 & 6 & 9 \\
\hline
\end{tabular}

intensities only assuming that the atomic data and/or the observations are in error by such an amount, an enhancement of a factor of 2 might suggest the need to review the atomic data used in the previous work and to perform new observations.

The scope of this paper is to give a new estimate of helium enhancement factors, using the differential emission measure (DEM) method (Lanzafame et al. 2002, 2005) as the main diagnostic tool, with the support of non-LTE calculations to include the opacity effects. From the observational point of view, an appropriate study of the EUV helium lines requires the joint use of SUMER and CDS on board SOHO and the EIS on board Hinode. The advantage in performing observations using these three instruments is that together they cover a large range of temperatures, from the upper chromosphere to the corona. The new observational sequences designed for this project and the selection of the lines used in the DEM analysis are described in Section 2. From the theoretical point of view, this work has been taken as a starting point for a complete update of atomic data, involved in the DEM technique, as discussed in Section 3. In Section 4 the DEM analysis and non-LTE calculations performed on the observational data taken from SUMER, CDS, and EIS are exploited, and the new helium enhancement factors are provided. Section 5 draws conclusions and final discussions.

\section{OBSERVATIONS}

The data examined in this work were taken during a joint observation campaign in 2009, on April 17 from 12:00 to 16:00 UT. These observations involved SOHO/SUMER (Wilhelm et al. 1995), SOHO/CDS Normal Incidence Spectrometer (NIS) (Harrison et al. 1995), and Hinode/EIS (Culhane et al. 2007) and were obtained under Joint Observing Program 220 and Hinode Operation Plan 109. The target was a quietSun area near the Sun center at $\left(0^{\prime \prime},-40^{\prime \prime}\right)$. Table 1 provides the main details of the joint observations. Line intensities were averaged over a common area observed by the three instruments to reduce substantially the effects of small-scale and short time fluctuations. Two raster maps were made with SUMER: the first scan includes selected bands with wavelengths shorter than the hydrogen Ly $\alpha$ line $\left(\lambda_{\mathrm{Ly} \alpha}=1215.7 \AA\right)$, while the second scan includes the wavelength ranges greater than $\lambda_{\text {Ly } \alpha}$. Six CDS and nine EIS scans were obtained. Each scan contains the whole set of wavelength bands, selected for the CDS and EIS observation sequences. To mitigate the effects of the lack of completely co-temporal observations, all CDS and EIS rasters were averaged and then analyzed with the two SUMER rasters.
The raw SUMER, CDS, and EIS data were reduced and calibrated using the standard procedures and routines within SolarSoft $^{5}$ (Brooks et al. 1999; Curdt et al. 2001; Lang et al. 2002, 2007; Young et al. 2009). The burn-in correction of the CDS/NIS detectors provided by del Zanna et al. (2010) was adopted in the present work. The uncertainty in the observed intensity for SUMER and CDS was estimated to be between $15 \%$ and $30 \%$, with an additional $10 \%$ error that was introduced for observations taken after SOHO's loss. For EIS the accuracy of the absolute intensity calibration was estimated to be around $20 \%$ before the launch (Lang et al. 2006). The pointing accuracy of SUMER and CDS is within $5^{\prime \prime}$, while typical values of EIS pointing uncertainty are $5^{\prime \prime}-10^{\prime \prime}$. However, the fields of view covered by the three spectrometers are wide enough to allow a good overlap and to perform an appropriate co-alignment (Giunta et al. 2012).

\subsection{Observation Sequences}

New observation sequences have been written for the three spectrometers. They were designed to ensure the spatial and temporal overlap between the instruments and to cover the wavelength range needed to study the EUV helium line intensities and perform an accurate DEM analysis. Therefore, the choice of the lines was done according to the following criteria: (1) lines possibly free from blends covering a large temperature range; (2) lines for density diagnostic, such as lines from $\mathrm{O} v$ observed by SUMER and CDS, and from Si VII and Fe XII observed by EIS; (3) EUV helium lines, observed mainly by CDS and EIS and in the second order of SUMER; (4) lines formed at low temperature $(\log T(\mathrm{~K}) \leqslant 4.5)$, such as lines from Si II and C II, observed by SUMER; (5) lines formed at medium $(4.5 \leqslant \log T(\mathrm{~K}) \leqslant 6.0)$ and higher $(\log T(\mathrm{~K})$ $\geqslant 6.0$ ) temperatures, to investigate a possible connection between the different layers of the solar atmosphere, such as lines from $\mathrm{O}$ III and $\mathrm{Ne} v$, observed by CDS, and from Fe $\mathrm{x}$ and Fe XII, observed by EIS; (6) lines for co-alignment and crosscalibration, such as lines observed by all three instruments, when possible, or which arise from the same ions or are characterized by similar temperatures of formation (e.g., $\mathrm{O}$ IV and $\mathrm{Ov}$ ).

The full list of lines used for this study is shown in Tables 2-4.

A scheme of the helium lines observed by CDS and EIS and the terms involved in the transitions is provided in Figure 1.

CDS allows observation of neutral helium EUV resonance lines, $1 \mathrm{~s}^{2}{ }^{1} \mathrm{~S}_{0}-1 \mathrm{~s} n \mathrm{p}{ }^{1} \mathrm{P}_{1}$ with $n=2,3,4$, 5, at wavelengths 584.33, 537.03, 522.21, and 515.62 A, respectively. The line at $584.33 \AA$ is also observed by SUMER in second order.

The doublet of He II at $303.78 \AA$ is observed by CDS in second order. It is a blend that arises from the transitions $1 \mathrm{~s}{ }^{2} \mathrm{~S}_{1 / 2}-2 \mathrm{p}{ }^{2} \mathrm{P}_{1 / 2,3 / 2}$. The resonance doublet of singly ionized helium, which involves the term $3 \mathrm{p}^{2} \mathrm{P}$, is observed by EIS at $256.32 \AA$. This doublet is complicated by a blend with several coronal lines, mainly Si x 256.37, Fe x 256.41 A, and Fe XIII $256.42 \AA$. However, for disk observations, He II has been estimated to contribute $70 \%-80 \%$ to the blend (Young et al. 2009; Giunta et al. 2012).

Furthermore, the intercombination multiplet of neutral helium between the terms $1 \mathrm{~s}^{2}{ }^{1} \mathrm{~S}$ and $1 \mathrm{~s} 2 \mathrm{p}{ }^{3} \mathrm{P}$ at $591.41 \AA$ is observed by CDS. It was identified, for the first time, by

http://lmsal.com/solarsoft/ 
Table 2

SUMER Spectral Lines Included in the Observations

\begin{tabular}{|c|c|c|c|}
\hline Instrument & Ion & $\lambda(\AA)$ & Transition \\
\hline SUMER & $\mathrm{C}_{\mathrm{II}}$ & 1335.71 & $2 \mathrm{~s}^{2} 2 \mathrm{p}{ }^{2} \mathrm{P}_{3 / 2}-2 \mathrm{~s} 2 \mathrm{p}^{2}{ }^{2} \mathrm{D}_{5 / 2}$ \\
\hline SUMER & $\mathrm{C}_{\mathrm{II}}$ & 1334.53 & $2 \mathrm{~s}^{2} 2 \mathrm{p}^{2} \mathrm{P}_{1 / 2}-2 \mathrm{~s} 2 \mathrm{p}^{2}{ }^{2} \mathrm{D}_{3 / 2}$ \\
\hline SUMER (i) & Si II & 1309.28 & $3 s^{2} 3 p{ }^{2} P_{3 / 2}-3 s 3 p^{2}{ }^{2} S_{1 / 2}$ \\
\hline SUMER & Si II & 1304.37 & $3 s^{2} 3 p{ }^{2} P_{1 / 2}-3 s 3 p^{2}{ }^{2} S_{1 / 2}$ \\
\hline SUMER & $\mathrm{Si}$ II & 1265.00 & $3 s^{2} 3 p{ }^{2} P_{3 / 2}-3 s^{2} 3 d^{2} D_{3 / 2}$ \\
\hline SUMER & Si II & 1264.74 & $3 s^{2} 3 p{ }^{2} P_{3 / 2}-3 s^{2} 3 d^{2} D_{5 / 2}$ \\
\hline SUMER & Si II & 1190.42 & $3 s^{2} 3 p^{2} \mathrm{P}_{1 / 2}-3 s 3 p^{2}{ }^{2} \mathrm{P}_{3 / 2}$ \\
\hline SUMER & S III & 1190.17 & $3 \mathrm{~s}^{2} 3 \mathrm{p}^{2}{ }^{3} \mathrm{P}_{0}-3 \mathrm{~s} 3 \mathrm{p}^{3}{ }^{3} \mathrm{D}_{1}$ \\
\hline SUMER & $\mathrm{C}$ III & 1176.37 & $2 \mathrm{~s} 2 \mathrm{p}{ }^{3} \mathrm{P}_{2}-2 \mathrm{p}^{2}{ }^{3} \mathrm{P}_{1}$ \\
\hline SUMER & $\mathrm{C}$ III & 1175.26 & $2 \mathrm{~s} 2 \mathrm{p}{ }^{3} \mathrm{P}_{0}-2 \mathrm{p}^{2}{ }^{3} \mathrm{P}_{1}$ \\
\hline SUMER (i) & $\mathrm{C}$ III & 1174.93 & $2 \mathrm{~s} 2 \mathrm{p}{ }^{3} \mathrm{P}_{1}-2 \mathrm{p}^{2}{ }^{3} \mathrm{P}_{2}$ \\
\hline SUMER & $\mathrm{S}_{\text {III }}$ & 1077.14 & $3 s^{2} 3 p^{2}{ }^{1} D_{2}-3 s^{2} 3 p 3 d^{1} D_{2}$ \\
\hline SUMER & $b \mathrm{O}$ IV & 790.19 & $2 s^{2} 2 p^{2} P_{3 / 2}-2 s 2 p^{2}{ }^{2} D_{5 / 2}$ \\
\hline SUMER & $b \mathrm{O}$ IV & 790.11 & $2 \mathrm{~s}^{2} 2 \mathrm{p}^{2} \mathrm{P}_{3 / 2}-2 \mathrm{~s} 2 \mathrm{p}^{2}{ }^{2} \mathrm{D}_{3 / 2}$ \\
\hline SUMER & $\mathrm{Na}$ VIII & 789.78 & $2 \mathrm{~s}^{2}{ }^{1} \mathrm{~S}_{0}-2 \mathrm{~s} 2 \mathrm{p}{ }^{3} \mathrm{P}_{1}$ \\
\hline SUMER (i) & O IV & 787.72 & $2 \mathrm{~s}^{2} 2 \mathrm{p}^{2} \mathrm{P}_{1 / 2}-2 \mathrm{~s} 2 \mathrm{p}^{2}{ }^{2} \mathrm{D}_{3 / 2}$ \\
\hline SUMER & $\mathrm{Ne}$ VIII & 780.30 & $2 \mathrm{~s}{ }^{2} \mathrm{~S}_{1 / 2}-2 \mathrm{p}{ }^{2} \mathrm{P}_{1 / 2}$ \\
\hline SUMER & $\mathrm{Ne}$ VIII & 770.42 & $2 \mathrm{~s}{ }^{2} \mathrm{~S}_{1 / 2}-2 \mathrm{p}{ }^{2} \mathrm{P}_{3 / 2}$ \\
\hline SUMER & Mg VIII & 762.65 & $2 s^{2} 2 p^{2} P_{1 / 2}-2 s 2 p^{2}{ }^{4} P_{3 / 2}$ \\
\hline SUMER & $\mathrm{Ov}$ & 761.99 & $2 \mathrm{~s} 2 \mathrm{p}{ }^{3} \mathrm{P}_{2}-2 \mathrm{p}^{2}{ }^{3} \mathrm{P}_{1}$ \\
\hline SUMER & $\mathrm{Ov}$ & 761.13 & $2 \mathrm{~s} 2 \mathrm{p}^{3} \mathrm{P}_{1}-2 \mathrm{p}^{2}{ }^{3} \mathrm{P}_{0}$ \\
\hline SUMER* & $\mathrm{Ov}$ & 629.73 & $2 \mathrm{~s}^{2}{ }^{1} \mathrm{~S}_{0}-2 \mathrm{~s} 2 \mathrm{p}{ }^{1} \mathrm{P}_{1}$ \\
\hline SUMER* & $\mathrm{He} \mathrm{I}$ & 584.33 & $1 \mathrm{~s}^{2}{ }^{1} \mathrm{~S}_{0}-1 \mathrm{~s} 2 \mathrm{p}{ }^{1} \mathrm{P}_{1}$ \\
\hline
\end{tabular}

Note. The symbol * indicates that the line is observed in the second order, while (i) specifies the lines used for the integral inversion (Section 4.1). The prefix $b$ designates the observed blend.

Brooks et al. (1999). They analyzed the measured and predicted line ratios between the $n=2$ and $n=5$ members of the $1 s^{2}{ }^{1} S-1 s n p{ }^{1} P$ series of $\mathrm{He} \mathrm{I}$ and the intercombination line and estimated them both in the optically thin approximation and in the optically thick case, using the non-LTE radiative transfer code MULTI. They found that the optically thin ratio $I$ $(591 \AA) / I(584 \AA)$ was too low compared with the observations, while the optically thick ratios were much closer to the observations, as expected for the $584.33 \AA$ line being affected by opacity and the $591.41 \AA$ line remaining optically thin, because of its very low radiative transition probability.

The He I $591.41 \AA$ intercombination line observed by CDS is the only optically thin helium line in the CDS and EIS spectra and can help to investigate the anomalous behavior of helium lines without including opacity effects. If the helium problem involves some processes that preferentially enhance the helium line intensities with respect to the other characteristic transition region lines, the same effect should be present in the intercombination line.

Furthermore, any spectral analysis based on DEM requires caution in many aspects, first of all the choice of lines used in the integral inversion. The main criteria for the selection of suitable lines are the following: (a) free from blends; (b) optically thin; (c) density insensitive; (d) corresponding accurate atomic data; (e) large temperature range coverage. Such criteria give critical constraints to the number of lines for the integral inversion. Therefore, for some lines (e.g., Si II, C II, and $\mathrm{C}_{\text {III }}$ ) it was necessary to make appropriate adjustments (Section 4.1.2) to satisfy the above requirements. The lines used for the integral inversion are marked by (i) in Tables $2-4$.
Table 3

CDS Spectral Lines Included in the Observations

\begin{tabular}{|c|c|c|c|}
\hline Instrument & Ion & $\lambda(\AA)$ & Transition \\
\hline CDS (i) & $\mathrm{Ov}$ & 629.73 & $2 \mathrm{~s}^{2}{ }^{1} \mathrm{~S}_{0}-2 \mathrm{~s} 2 \mathrm{p}{ }^{1} \mathrm{P}_{1}$ \\
\hline CDS (i) & $\mathrm{O}_{\text {III }}$ & 599.60 & $2 \mathrm{~s}^{2} 2 \mathrm{p}^{2}{ }^{1} \mathrm{D}_{2}-2 \mathrm{~s} 2 \mathrm{p}^{3}{ }^{1} \mathrm{D}_{2}$ \\
\hline CDS & $\mathrm{O}_{\text {III }}$ & 597.82 & $2 \mathrm{~s}^{2} 2 \mathrm{p}^{2}{ }^{1} \mathrm{~S}_{0}-2 \mathrm{~s} 2 \mathrm{p}^{3}{ }^{1} \mathrm{P}_{1}$ \\
\hline CDS & $b \mathrm{He}_{\mathrm{I}}$ & 591.41 & $1 \mathrm{~s}^{2}{ }^{1} \mathrm{~S}_{0}-1 \mathrm{~s} 2 \mathrm{p}{ }^{3} \mathrm{P}_{2}$ \\
\hline CDS & $b \mathrm{He}_{\mathrm{I}}$ & 591.41 & $1 \mathrm{~s}^{2}{ }^{1} \mathrm{~S}_{0}-1 \mathrm{~s} 2 \mathrm{p}{ }^{3} \mathrm{P}_{1}$ \\
\hline CDS & Ar VII & 585.75 & $3 s^{2}{ }^{1} S_{0}-3 s 3 p^{1} P_{1}$ \\
\hline CDS & $\mathrm{He}_{\mathrm{I}}$ & 584.33 & $1 \mathrm{~s}^{2}{ }^{1} \mathrm{~S}_{0}-1 \mathrm{~s} 2 \mathrm{p}{ }^{1} \mathrm{P}_{1}$ \\
\hline CDS & $\mathrm{Cax}$ & 574.01 & $3 \mathrm{~s}^{2} \mathrm{~S}_{1 / 2}-3 \mathrm{p}{ }^{2} \mathrm{P}_{1 / 2}$ \\
\hline CDS & O IV & 555.76 & $2 s^{2} 2 p^{2} P_{3 / 2}-2 s 2 p^{2}{ }^{2} P_{1 / 2}$ \\
\hline CDS & O IV & 554.51 & $2 s^{2} 2 p^{2} \mathrm{P}_{3 / 2}-2 s 2 p^{2}{ }^{2} \mathrm{P}_{3 / 2}$ \\
\hline CDS & O IV & 554.08 & $2 s^{2} 2 p^{2} \mathrm{P}_{1 / 2}-2 s 2 p^{2}{ }^{2} \mathrm{P}_{1 / 2}$ \\
\hline CDS & O IV & 553.33 & $2 \mathrm{~s}^{2} 2 \mathrm{p}{ }^{2} \mathrm{P}_{1 / 2}-2 \mathrm{~s} 2 \mathrm{p}^{2}{ }^{2} \mathrm{P}_{3 / 2}$ \\
\hline CDS (i) & $\mathrm{Ne}$ IV & 543.88 & $2 s^{2} 2 p^{3}{ }^{4} S_{3 / 2}-2 s 2 p^{4}{ }^{4} P_{5 / 2}$ \\
\hline CDS & $\mathrm{Ne}$ IV & 542.07 & $2 s^{2} 2 p^{3}{ }^{4} S_{3 / 2}-2 s 2 p^{4}{ }^{4} P_{3 / 2}$ \\
\hline CDS & $b \mathrm{O}_{\mathrm{II}}$ & 538.32 & $2 \mathrm{~s}^{2} 2 \mathrm{p}^{3}{ }^{2} \mathrm{D}_{3 / 2}-2 \mathrm{~s} 2 \mathrm{p}^{4}{ }^{2} \mathrm{P}_{3 / 2}$ \\
\hline CDS & $b \mathrm{C}$ III & 538.31 & $2 s 2 p{ }^{3} P_{2}-2 s 3 s{ }^{3} S_{1}$ \\
\hline CDS & $b \mathrm{O}$ II & 538.26 & $2 \mathrm{~s}^{2} 2 \mathrm{p}^{3}{ }^{2} \mathrm{D}_{5 / 2}-2 \mathrm{~s} 2 \mathrm{p}^{4}{ }^{2} \mathrm{P}_{3 / 2}$ \\
\hline CDS & $b \mathrm{C}$ III & 538.15 & $2 \mathrm{~s} 2 \mathrm{p}{ }^{3} \mathrm{P}_{1}-2 \mathrm{~s} 3 \mathrm{~s}^{3} \mathrm{~S}_{1}$ \\
\hline CDS & $b \mathrm{C}$ III & 538.08 & $2 \mathrm{~s} 2 \mathrm{p}{ }^{3} \mathrm{P}_{0}-2 \mathrm{~s} 3 \mathrm{~s}{ }^{3} \mathrm{~S}_{1}$ \\
\hline CDS & $b \mathrm{O}_{\mathrm{II}}$ & 537.83 & $2 \mathrm{~s}^{2} 2 \mathrm{p}^{3}{ }^{2} \mathrm{D}_{3 / 2}-2 \mathrm{~s} 2 \mathrm{p}^{4}{ }^{2} \mathrm{P}_{1 / 2}$ \\
\hline CDS & $\mathrm{He}$ I & 537.03 & $1 \mathrm{~s}^{2}{ }^{1} \mathrm{~S}_{0}-1 \mathrm{~s} 3 \mathrm{p}{ }^{1} \mathrm{P}_{1}$ \\
\hline CDS & $\mathrm{O}_{\text {III }}$ & 525.80 & $2 \mathrm{~s}^{2} 2 \mathrm{p}^{2}{ }^{1} \mathrm{D}_{2}-2 \mathrm{~s} 2 \mathrm{p}^{3}{ }^{1} \mathrm{P}_{1}$ \\
\hline CDS & $\mathrm{He}_{\mathrm{I}}$ & 522.21 & $1 \mathrm{~s}^{2}{ }^{1} \mathrm{~S}_{0}-1 \mathrm{~s} 4 \mathrm{p}{ }^{1} \mathrm{P}_{1}$ \\
\hline CDS (i) & Si XII & 520.66 & $2 \mathrm{~s}^{2} \mathrm{~S}_{1 / 2}-2 \mathrm{p}{ }^{2} \mathrm{P}_{1 / 2}$ \\
\hline CDS & $\mathrm{He}_{\mathrm{I}}$ & 515.62 & $1 \mathrm{~s}^{2}{ }^{1} \mathrm{~S}_{0}-1 \mathrm{~s} 5 \mathrm{p}{ }^{1} \mathrm{P}_{1}$ \\
\hline CDS* & $b \mathrm{He}$ II & 303.79 & $1 \mathrm{~s}{ }^{2} \mathrm{~S}_{1 / 2}-2 \mathrm{p}{ }^{2} \mathrm{P}_{1 / 2}$ \\
\hline $\mathrm{CDS}^{*}$ & $b \mathrm{He}$ II & 303.78 & $1 \mathrm{~s}{ }^{2} \mathrm{~S}_{1 / 2}-2 \mathrm{p}{ }^{2} \mathrm{P}_{3 / 2}$ \\
\hline CDS* & Si XI & 303.33 & $2 \mathrm{~s}^{2}{ }^{1} \mathrm{~S}_{0}-2 \mathrm{~s} 2 \mathrm{p}{ }^{1} \mathrm{P}_{1}$ \\
\hline
\end{tabular}

Note. For the symbols *, (i), and $b$ see Table 2.

Table 4

EIS Spectral Lines Included in the Observations

\begin{tabular}{|c|c|c|c|}
\hline Instrument & Ion & $\lambda(\AA)$ & Transition \\
\hline EIS & O IV & 279.93 & $2 s^{2} 2 p^{2} P_{3 / 2}-2 s^{2} 3 s^{2} S_{1 / 2}$ \\
\hline EIS & O IV & 279.63 & $2 s^{2} 2 p^{2} P_{1 / 2}-2 s^{2} 3 s^{2} S_{1 / 2}$ \\
\hline EIS (i) & Si VII & 275.67 & $2 \mathrm{~s}^{2} 2 \mathrm{p}^{4}{ }^{3} \mathrm{P}_{1}-2 \mathrm{~s} 2 \mathrm{p}^{5}{ }^{3} \mathrm{P}_{1}$ \\
\hline EIS & Si VII & 275.35 & $2 \mathrm{~s}^{2} 2 \mathrm{p}^{4}{ }^{3} \mathrm{P}_{2}-2 \mathrm{~s} 2 \mathrm{p}^{5}{ }^{3} \mathrm{P}_{2}$ \\
\hline EIS & $b \mathrm{Fe}$ XIII & 256.42 & $3 s^{2} 3 p^{2}{ }^{1} D_{2}-3 s 3 p^{3}{ }^{1} P_{1}$ \\
\hline EIS & $b \mathrm{Fe} \times$ & 256.41 & $3 s^{2} 3 p^{5}{ }^{2} P_{3 / 2}-3 s^{2} 3 p^{4} 3 d^{4} D_{3 / 2}$ \\
\hline EIS & $b \mathrm{Six}$ & 256.37 & $2 \mathrm{~s}^{2} 2 \mathrm{p}^{2} \mathrm{P}_{1 / 2}-2 \mathrm{~s} 2 \mathrm{p}^{2}{ }^{2} \mathrm{P}_{1 / 2}$ \\
\hline EIS & $b \mathrm{He}$ II & 256.32 & $1 \mathrm{~s}{ }^{2} \mathrm{~S}_{1 / 2}-3 \mathrm{p}{ }^{2} \mathrm{P}_{3 / 2}$ \\
\hline EIS & $b \mathrm{He}$ II & 256.32 & $1 \mathrm{~s}{ }^{2} \mathrm{~S}_{1 / 2}-3 \mathrm{p}{ }^{2} \mathrm{P}_{1 / 2}$ \\
\hline EIS (i) & $\mathrm{Fe} \mathrm{XII}$ & 193.51 & $3 s^{2} 3 p^{3}{ }^{4} S_{3 / 2}-3 s^{2} 3 p^{2} 3 d{ }^{4} P_{3 / 2}$ \\
\hline EIS & $b \mathrm{O}$ IV & 186.88 & $2 \mathrm{~s} 2 \mathrm{p}^{2}{ }^{4} \mathrm{P}_{1 / 2}-2 \mathrm{p}^{2} 3 \mathrm{p}{ }^{4} \mathrm{P}_{1 / 2}$ \\
\hline EIS & $b \mathrm{Fe} \mathrm{XII}$ & 186.88 & $3 s^{2} 3 p^{3}{ }^{2} D_{5 / 2}-3 s^{2} 3 p^{2} 3 d^{2} F_{7 / 2}$ \\
\hline EIS & $b \mathrm{O}$ IV & 186.86 & $2 \mathrm{~s} 2 \mathrm{p}^{2}{ }^{4} \mathrm{P}_{1 / 2}-2 \mathrm{p}^{2} 3 \mathrm{p}{ }^{4} \mathrm{P}_{3 / 2}$ \\
\hline EIS & $b \mathrm{Fe} \mathrm{XII}$ & 186.85 & $3 s^{2} 3 p^{3}{ }^{2} D_{3 / 2}-3 s^{2} 3 p^{2} 3 d^{2} F_{5 / 2}$ \\
\hline EIS (i) & Fe VIII & 186.60 & $3 p^{6} 3 d^{2} D_{3 / 2}-3 p^{5} 3 d^{2}{ }^{2} F_{5 / 2}$ \\
\hline EIS (i) & $\mathrm{O}$ VI & 184.12 & $2{ }^{2} P_{3 / 2}-3 s^{2} S_{1 / 2}$ \\
\hline EIS & $\mathrm{O}$ vI & 183.94 & $2 \mathrm{p}^{2} \mathrm{P}_{1 / 2}-3 \mathrm{~s}^{2} \mathrm{~S}_{1 / 2}$ \\
\hline
\end{tabular}

Note. For the symbols *, (i), and $b$ see Table 2. 


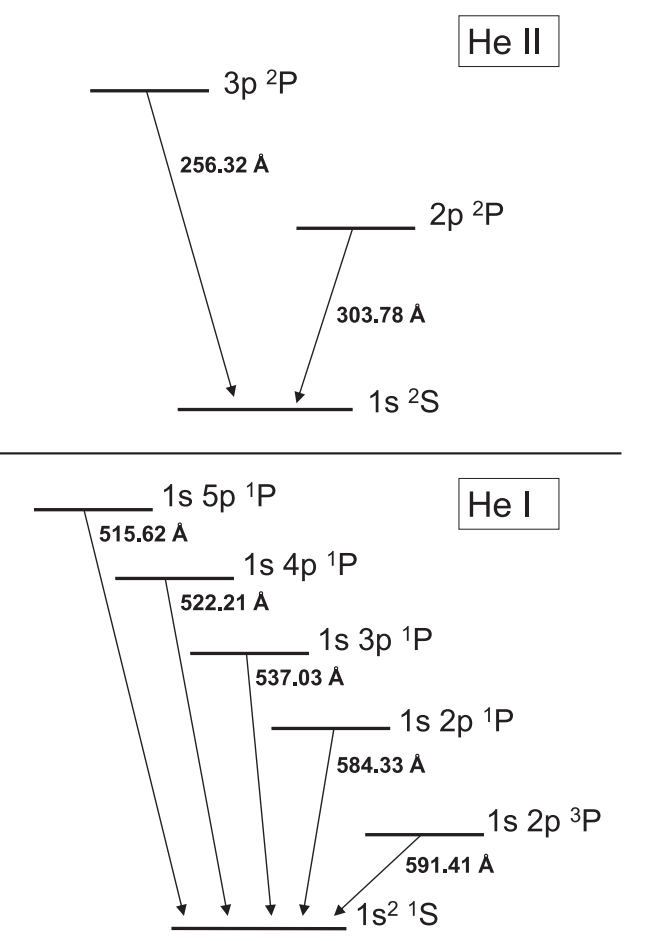

Figure 1. Partial level diagram for $\mathrm{He}$ I and $\mathrm{He}$ II.

For SUMER and CDS, the numerous spectral analyses performed in the previous literature (Brooks et al. 1999; Curdt et al. 2001; Lanzafame et al. 2005) were used as a starting point for line and blend identification and selection. For EIS the work of Young et al. (2007) has been adopted as a reference to identify line blends and lines used for density diagnostics (e.g., $\mathrm{Si}$ VII and Fe XII).

\section{ATOMIC DATA AND MODELING}

A realistic examination of helium line behavior requires an accurate model of atomic populations not only of helium ions but also of the other ions that are included in the DEM analysis. Particular attention has been given to low-charge silicon ions. Si II multiplets, observed by SUMER (Table 2), arise at the low temperatures $(\log T(\mathrm{~K}) \leqslant 4.5)$ at which helium lines are formed, providing an essential diagnostic tool. These lines are formed in the upper chromosphere/lower transition region (Lanzafame 1994), where the density effects are not negligible and the usual coronal zero-density model is not appropriate. Therefore, the generalized collisional-radiative (GCR) theory (Summers et al. 2006) is the most suitable approach in this context. The method to derive the DEM and its relationship with the GCR theory has been introduced and described in detail by Lanzafame et al. (2002) and in the Atomic Data and Analysis Structure (ADAS) manual (Summers 2001). Following the Lanzafame et al. (2002) approach, the intensity of a spectral line, which originates from a transition between an upper level $j$ and a lower level $k$, is written as

$$
I_{j \rightarrow k}=\frac{\mathcal{A}(Z)}{4 \pi} \int G_{j \rightarrow k}\left(T_{\mathrm{e}}\right) \Phi\left(T_{\mathrm{e}}\right) d T_{\mathrm{e}},
$$

where $\mathcal{A}(Z)$ is the abundance of the element, $G_{j \rightarrow k}\left(T_{\mathrm{e}}\right)$ is the contribution function, and $\Phi\left(T_{\mathrm{e}}\right)$ is the DEM. The contribution function is formed by two main terms, which take into account the atomic population of excited levels and the ionization fraction. Although in Equation (1) the contribution function is written as a function of electron temperature alone, it depends also on electron density, and so the relationship between these two variables $\left(T_{\mathrm{e}}\right.$ and $\left.N_{\mathrm{e}}\right)$ must be taken into account. Two common assumptions are that $N_{\mathrm{e}}$ or the electron pressure $P_{\mathrm{e}} \sim T_{\mathrm{e}} N_{\mathrm{e}}$ is constant over the range of temperature where the $G$ function has significant values (Section 4).

In the following subsections the data adopted to build up the contribution functions used for this work are discussed. They are calculated using ADAS codes and fundamental data from the ADAS database. Comparison with fundamental data from the literature is noted where appropriate.

\subsection{Energy Levels, Radiative and Collisional Data}

\subsubsection{H-like Ions}

For $\mathrm{He}^{1+}$ ions the energy values of terms up to $5 g$ are taken from the National Institute of Standards and Technology (NIST), ${ }^{6}$ as well as the radiative transition probabilities for transitions between the terms $1 \mathrm{~s}{ }^{2} \mathrm{~S}-2 \mathrm{p}{ }^{2} \mathrm{P}, 1 \mathrm{~s}{ }^{2} \mathrm{~S}-3 \mathrm{p}{ }^{2} \mathrm{P}$, and $2 \mathrm{p}{ }^{2} \mathrm{P}-5 \mathrm{~d}{ }^{2} \mathrm{D}$. The most recent collision strength data are the $R$-matrix with pseudo-states calculations of Ballance et al. (2003) and are adopted here. However, because of the degeneracy, terms with the same principal quantum number $n$ have been grouped ( $b n$ resolution).

\subsubsection{He-like Ions}

Along the He-like iso-electronic sequence, only lines from neutral helium are selected for this work. As mentioned in Section 2, these lines are all the EUV resonance lines between the ground term $1 \mathrm{~s}^{2}{ }^{1} \mathrm{~S}$ and the excited terms $1 \mathrm{~s} n \mathrm{p}^{1} \mathrm{P}$, with $n=2,3,4,5$ and the intercombination line between the $1 \mathrm{~s}^{2}{ }^{1} \mathrm{~S}$ in the singlet state and the $1 \mathrm{~s} 2 \mathrm{p}{ }^{3} \mathrm{P}$ term in the triplet state. The $l s$ resolution is a good approximation for $\mathrm{He}^{0+}$, because levels belonging to the same term are close to degenerate. The first 29 energy terms up to $5 \mathrm{p}^{1} \mathrm{P}$ have been included in the model. Energy levels come from the NIST database. The radiative transition probability for the transition $1 \mathrm{~s}^{2}{ }^{1} \mathrm{~S}-1 \mathrm{~s} 2 \mathrm{p}{ }^{3} \mathrm{P}$ has been taken from Lach \& Pachucki (2001). For the configurations up to 1s4f, the data were assembled by Paton (2005), including earlier surveys of de Heer et al. (1992 p. 47) and $R$ matrix calculation of Ballance et al. (2003). Plane wave Born (PWB) calculations, using the Cowan code (Cowan 1981), have been performed to add transitions that involve $n=5$ configurations and then merged with the more accurate $R$ matrix calculations.

\subsubsection{Li-like Ions}

The observed lines, belonging to the Li-like sequence, arise from transitions between the ground level $1 \mathrm{~s}^{2} 2 \mathrm{~s}^{2} \mathrm{~S}_{1 / 2}$ and the fine-structure levels $1 \mathrm{~s}^{2} 2 \mathrm{p}^{2} \mathrm{P}_{1 / 2,3 / 2}$ of the ions $\mathrm{Ne}^{7+}$ and $\mathrm{Si}^{11+}$ (SUMER and CDS) and from transitions between these latter excited levels and the level $1 \mathrm{~s}^{2} 3 \mathrm{~s}^{2} \mathrm{~S}_{1 / 2}$ of the ion $\mathrm{O}^{5+}$ (EIS). The energy levels, A-values, and collision strengths of Zhang et al. (1990) are adopted. The data include fine-structure resolved levels up to $5 g^{2} G_{9 / 2}$. The relativistic distorted wave effective collision strengths of Zhang et al. (1990) were

\footnotetext{
6 http://www.nist.gov/
} 
modified to correct the data for the effect of resonances and to increase or decrease their values to bring them into closer agreement with the close-coupling data, as reviewed by McWhirter (1994).

\subsubsection{Be-like Ions}

The required ions of Be-like system are $\mathrm{O}^{4+}, \mathrm{C}^{2+}, \mathrm{Na}^{7+}$, and $\mathrm{Si}^{10+}$. The most important transitions arising from $\mathrm{O}^{4+}$ are $2 \mathrm{~s}^{2}{ }^{1} \mathrm{~S}_{0}-2 \mathrm{~s} 2 \mathrm{p}{ }^{1} \mathrm{P}_{1}$, which gives rise to the resonance line at $629.73 \AA$ (observed both by SUMER in second order and by CDS), and $2 \mathrm{~s} 2 \mathrm{p}{ }^{3} \mathrm{P}_{1}-2 \mathrm{p}^{2}{ }^{3} \mathrm{P}_{2}$, which gives rise to the line at $762.00 \AA$ (observed by SUMER). Their ratio will be used as a density diagnostic in Section 4. Also, the $2 s 2 p{ }^{3} P-2 s 3 d{ }^{3} D$ multiplet is observed by EIS. Transitions between the terms $2 s 2{ }^{3} \mathrm{P}-2 \mathrm{p}^{2}{ }^{3} \mathrm{P}$ from $\mathrm{C}^{2+}$ are also relevant. For $\mathrm{Na}^{7+}$, only the intercombination line between the levels $2 s^{2}{ }^{1} \mathrm{~S}_{0}-2 \mathrm{~s} 2 \mathrm{p}{ }^{3} \mathrm{P}_{1}$ is present in the current observations. It is a very faint line that lies in the wing of the more intense line of $\mathrm{O}^{3+}$ at around $790 \AA$. For these Be-like ions, the first 20 levels up to $2 \mathrm{~s} 3 \mathrm{~d}^{1} \mathrm{D}_{2}$ have been included in the data. For $\mathrm{O}^{4+}$ and $\mathrm{C}^{2+}$ the energy levels come from Moore (1993). Radiative transition probabilities from Hibbert (1980) have been adopted for $\mathrm{O}^{4+}$, while the main source for $\mathrm{C}^{2+}$ is Allard et al. (1990). For $\mathrm{Na}^{7+}$ both the energy levels and A-values have been taken from Zhang et al. (1990), except for the forbidden transitions, which come from Muehlethaler \& Nussbaumer (1976). The electron impact excitation data have been taken following the recommendations of Berrington (1994). For $\mathrm{Si}^{10+}$, energy levels come from NIST, where available, and from the calculations of Bhatia \& Landi (2007). The collision strengths have been calculated by Berrington et al. (1985) using the $R$-matrix method for the first 10 levels, while A-values come from Muehlethaler \& Nussbaumer (1976). For the other 82 levels, belonging to the configurations $2 l 3 l^{\prime}, 2 l 4 l^{\prime}$, and $2 \mathrm{~s} 5 l^{\prime}$ with $l=\mathrm{s}, \mathrm{p}$ and $l^{\prime}=\mathrm{s}, \mathrm{p}$, $\mathrm{d}$, the data have been derived by Bhatia \& Landi (2007), using the distorted wave approximation. These data have been supplemented with PWB Cowan code calculations to add missing transitions.

\subsubsection{B-like Ions}

For $\mathrm{C}^{1+}$ and $\mathrm{Mg}^{7+}$, the first 18 and 15 fine-structure energy levels up to $2 \mathrm{p}^{3}{ }^{2} \mathrm{P}_{3 / 2}$ are included. The energy levels adopted for these two ions are from Edlén (1983). The radiative transition probabilities between the $1 / 2$ and $3 / 2$ levels of the ground terms have been derived from the multi-configuration Hartree-Fock results of Froese Fischer (1983), while for the other transitions the A-values of Merkelis et al. (1995) have been used. The effective collision strengths have been taken from the review of Sampson et al. (1994). For the $\mathrm{O}^{3+}$ ion, the data assessed by Giunta et al. (2012) have been used. For $\mathrm{Si}^{9+}$, only the line that arises from the transition between the ground level $2 s^{2} 2 p{ }^{2} \mathrm{P}_{1 / 2}$ and the excited level $2 \mathrm{~s} 2 \mathrm{p}^{2}{ }^{2} \mathrm{P}_{1 / 2}$ is present in the observations analyzed for this work. This line is observed in a blend with the strong resonance line of $\mathrm{He}^{1+}$ at $256.32 \AA$. Hence, a correct estimate of its absolute intensity is important to evaluate how much it contributes to the blend. New electron impact collision strength results have been reported by Liang et al. (2009). Their calculations have been performed using intermediate coupling frame transformation (ICFT; Griffin et al. 1998) $R$-matrix calculation and are adopted here, while the energy levels have been taken from NIST.

\subsubsection{C-like Ions}

The only C-like ion, included in the current observation sequences, is $\mathrm{O}^{2+}$. The first 46 fine-structure resolved energy levels (belonging to the configurations $2 \mathrm{~s}^{2} 2 \mathrm{p}^{2}, 2 \mathrm{~s} 2 \mathrm{p}^{3}$, and $2 \mathrm{~s}^{2} 2 \mathrm{p} 3 l$ with $l=\mathrm{s}, \mathrm{p}, \mathrm{d}$ ) have been included in the model. The energy levels come from NIST, while the transition probabilities come from the critical compilation of Wiese et al. (1996). The effective collision strengths have been computed by Aggarwal \& Keenan (1999), using the $R$-matrix method.

\subsubsection{N-like Ions}

CDS observes the multiplet that arises from transitions between the terms $2 \mathrm{~s}^{2} 2 \mathrm{p}^{3}{ }^{2} \mathrm{D}-2 \mathrm{~s} 2 \mathrm{p}^{4}{ }^{2} \mathrm{P}$ of $\mathrm{O}^{1+}$ blended with the multiplet $2 \mathrm{~s} 2 \mathrm{p}{ }^{3} \mathrm{P}-2 \mathrm{~s} 3 \mathrm{~s}{ }^{3} \mathrm{~S}$ of $\mathrm{C}^{2+}$ and two resolved lines (at 542.07 and $543.89 \AA$ ) of the multiplet $2 \mathrm{~s}^{2} 2 \mathrm{p}^{3}{ }^{4} S-2 \mathrm{~s} 2 \mathrm{p}^{4}{ }^{4} \mathrm{P}$ of $\mathrm{Ne}^{3+}$. For $\mathrm{O}^{1+} l s$ resolution is appropriate, while for $\mathrm{Ne}^{3+} i c$ resolution is required. Data for $\mathrm{O}^{1+}$ have been taken from the review of Brooks (1997) and supplemented with PWB Cowan calculations. The main source for effective collision strengths is McLaughlin \& Bell (1994). For $\mathrm{Ne}^{3+}$, the energy levels from NIST have been taken where available. The other values come from Cowan. Oscillator strengths of Bhatia \& Kastner (1988) have been adopted. The collision strengths are from Ramsbottom et al. (1998), calculated in the close-coupling approximation using the multichannel $R$-matrix method.

\subsubsection{O-like Ions}

Along the O-like sequence, the two lines that arise from $\mathrm{Si}^{6+}$, observed by EIS, are used in this work as density diagnostics. The atomic model for $\mathrm{Si}^{6+}$ includes 86 fine-structure energy levels up to $2 \mathrm{~s}^{2} 2 \mathrm{p}^{3} 3 \mathrm{~d}^{1} \mathrm{P}_{1}$ in intermediate-coupling. The excitation data available come from Bhatia \& Landi (2003), using the distorted wave approximation, and are supplemented by Cowan calculations for the missing transitions. Energy levels and A-values are taken from NIST where available. Alternatively, the Bhatia \& Landi (2003) and Cowan theoretical values are adopted.

\subsubsection{Na-like Ions}

For Na-like calcium, the first 21 fine-structure resolved levels up to $5 g{ }^{2} G_{9 / 2}$ are included in the model. The data for this sequence have been taken from the work of Sampson et al. (1990), who calculated collision data in the distorted wave approximation. The energy levels and A-values have been replaced by NIST values where possible.

\subsubsection{Mg-like Ions}

The data for $\mathrm{Ar}^{6+}$ come from the ICFT $R$-matrix closecoupling calculations of Griffin et al. (1999). The calculations include 25 terms and 45 levels belonging to the configurations $3 l^{2}, 3 \mathrm{~s} 3 l^{\prime}$, and $3 \mathrm{~s} 4 l^{\prime \prime}$ with $l=\mathrm{s}, \mathrm{p}, \mathrm{d}, l^{\prime}=\mathrm{p}, \mathrm{d}$, and $l^{\prime \prime}=\mathrm{s}, \mathrm{p}, \mathrm{d}$. The line at $585.75 \AA$ from this ion, observed by CDS, lies in the wing of the strong resonance line at $584.32 \AA$, which arises from neutral helium. The evaluation of its intensity is needed to avoid overestimating the helium line intensity. 


\subsubsection{Al-like Ions}

The calculations adopted here for $\mathrm{Si}^{1+}$ have been performed by Dufton \& Kingston (1991). They included in their model 15 fine-structure levels up to $3 \mathrm{~s} 3 \mathrm{p}^{2}{ }^{2} \mathrm{P}_{3 / 2}$ and calculated collision strengths using the $R$-matrix approach for transitions among the lowest seven levels and for transitions from the ground state $3 \mathrm{~s}^{2} 3 \mathrm{p}{ }^{2} \mathrm{P}_{j}$ (with $j=1 / 2,3 / 2$ ) to all the other levels. The energy levels come from NIST and radiative data from Nahar (1998) and Nussbaumer (1977). These data have been supplemented with Cowan calculations to add the higher levels belonging to the configurations $3 \mathrm{~s} 3 \mathrm{p} 3 \mathrm{~d}$ and $3 p^{3}$ and transitions among them.

\subsubsection{Si-like Ions}

The electron impact data for $\mathrm{S}^{2+}$ have been derived using AUTOSTRUCTURE (Badnell 1997) in the distorted wave approximation. For $\mathrm{Fe}^{12+}$, the rate coefficients for electron collision excitation are taken from the $R$-matrix calculation performed by Storey \& Zeippen (2010). The line at $256.42 \AA$ from this ion is observed in a blend with the much stronger resonance line arising from $\mathrm{He}^{1+}$, together with the line at $256.32 \AA$ from $\mathrm{Si}^{9+}$. The contribution of an ion such as $\mathrm{Fe}^{12+}$ is negligible in quiet-Sun conditions, but it may become significant in active regions.

\subsubsection{P-like, Cl-like, and K-like Iron Ions}

Lines that arise from $\mathrm{Fe}^{11+}, \mathrm{Fe}^{9+}$, and $\mathrm{Fe}^{7+}$ are at the shorter wavelength range that is covered by EIS. For $\mathrm{Fe}^{11+}$, the scattering calculation, performed by Storey et al. (2005) using the $R$-matrix method, includes the lowest $58 l s$ terms, which give rise to 143 fine-structure levels. The energy levels and Avalues come from del Zanna \& Mason (2005). These data have been merged with the PWB Cowan calculation to add missing levels belonging to the $3 \mathrm{~s}^{2} 3 \mathrm{p} 3 \mathrm{~d}^{2}$ and $3 \mathrm{~s} 3 \mathrm{p}^{3} 3 \mathrm{~d}$ configurations and the respective transitions. For $\mathrm{Fe}^{9+}$, energy levels, Avalues, and effective collision strengths up to 54 levels are from the review of del Zanna et al. (2004). For the other 118 levels the distorted wave approximation from the work of Malinovsky et al. (1980) is adopted. As for $\mathrm{Fe}^{11+}$, the data have been supplemented with PWB calculations to fill the gap left by the missing transitions. For the last iron ion, ICTF $R$-matrix closecoupling calculations of Griffin et al. (2000), which include 33 terms and 77 levels belonging to the configurations $3 p^{6} 3 d$, $3 \mathrm{p}^{5} 3 \mathrm{~d}^{2}, 3 \mathrm{p}^{5} 3 \mathrm{~d} 4 \mathrm{~s}, 3 \mathrm{p}^{6} 4 \mathrm{~s}, 3 \mathrm{p}^{6} 4 l$ with $l=\mathrm{p}, \mathrm{d}$, $\mathrm{f}$ in the closecoupling expansion, are used.

\subsection{Ionisation and Recombination Data}

Ionisation and recombination data for all the ions of helium, carbon, oxygen, and neon have been obtained following the GCR approach, as described by Summers et al. (2006). For the ions of magnesium, sodium, calcium, argon, and sulphur the ionization balance of Arnaud \& Rothenflug (1985) has been used, while for iron ions the Arnaud \& Raymond (1992) results have been adopted. The calculations of Arnaud \& Rothenflug (1985) and Arnaud \& Raymond (1992) have been scaled in $N_{\mathrm{e}}$, to take into account the effect of finite electron density (Summers 1974). For the silicon ions new calculations have been done, using a full GCR approach (http://open.adas.ac.uk).

\section{SPECTRAL ANALYSIS}

In this section, the application of the DEM technique to the observations described in Section 2 is discussed. Such an investigation is supported by further analyses, to derive electron density and pressure (Section 4.1.1) and elemental abundances (Section 4.1.3). The corrections adopted for the observed intensities of $\mathrm{Si}$ II, $\mathrm{C}_{\text {III }}$, and $\mathrm{C}_{\text {III }}$ lines are illustrated (Section 4.1.2). non-LTE radiative transfer calculations are also performed in Section 4.1.5. Finally, the results for helium enhancement are compared with the previous literature.

\subsection{Differential Emission Measure}

Many mathematical procedures have been developed to deduce the DEM in temperature by inverting Equation (1). The technique adopted for this work is the data adaptive smoothing approach of Thompson (1990), which has been implemented through the Glasgow code (Harrison \& Thompson 1991) and automated within ADAS, with the routine ADAS601. As mentioned, a full description of the method is provided by Lanzafame et al. (2002). However, the extrapolation at low electron temperature $\left(T<\min \left(T_{i}^{\max }\right)\right.$, where $\min \left(T_{i}^{\max }\right)$ is the temperature corresponding to the maximum of the $G$ function representing the coldest observed line) of the initial estimate of DEM, $\Phi_{0}(T)$, has been modified from a B-spline to linear, to avoid inconsistent interpretation in the region where helium lines are formed.

The input data for deriving the DEM are the set of observed intensities (Section 2), their contribution functions (Section 3), and the elemental abundances $\mathcal{A}(Z)$ (Equation (1)).

The lines used in the integral inversion have been listed in Tables 2-4 and marked by (i), while all the other lines are compared with the observations, using the DEM curve obtained in the present analysis.

Since the contribution functions are calculated under the two assumptions of constant electron density or constant electron pressure, an estimate of electron density and/or electron pressure from the observations is required to provide those values that give a satisfactory description of the observed plasma conditions. Once the electron density and pressure have been evaluated, the elemental abundance analysis is performed, adjusting the values to get the minimum deviation from observations.

\subsubsection{Electron Density and Electron Pressure Estimate}

A preliminary study using intensity ratios of lines emitted by the same ion is performed. Only three density-sensitive line ratios are not affected by known blends in the present observations. From an atomic point of view, the choice of these ratios is related to the density sensitivity of the population ratio, which can be due to the competing importance of collisional and radiative de-excitation from the upper level or to the dependence on density of the population of the lower levels from which the upper level is populated.

The first density-sensitive line ratio belongs to the Be-like system and involves the two $\mathrm{Ov}$ lines. The first excited configuration $2 \mathrm{~s} 2 \mathrm{p}$ results in a ${ }^{1} \mathrm{P}$ and a ${ }^{3} \mathrm{P}$ term. The ${ }^{1} \mathrm{P}$ term is excited from the ground state ${ }^{1} \mathrm{~S}$ and produces the strong resonance line at $629.73 \AA$, observed by both CDS and SUMER in second order. The $2 \mathrm{~s} 2 \mathrm{p}{ }^{3} \mathrm{P}$ term is also excited by the ground state, but it is metastable. As a consequence, lines related to collisional excitations from it are density sensitive. 
Table 5

Selected Density-sensitive Line Ratios for the Evaluation of the $G$ Functions

\begin{tabular}{lccrc}
\hline \hline Ion & Ratio $(\AA)$ & $\log T^{\text {peak }}$ & \multicolumn{1}{c}{$\log N_{\mathrm{e}}$} & $\log P_{\mathrm{e}}$ \\
\hline O v & $762.00 / 629.73$ & 5.40 & $10.0 \pm 0.2$ & $15.4 \pm 0.2$ \\
Si VII & $275.35 / 275.67$ & 5.75 & $9.4 \pm 0.3$ & $15.1 \pm 0.3$ \\
Fe XII & $193.51 / 186.88$ & 6.10 & $8.9 \pm 0.1$ & $15.0 \pm 0.1$ \\
\hline
\end{tabular}

Note. $T^{\text {peak }}$ is the peak temperature of line formation, $N_{\mathrm{e}}$ and $P_{\mathrm{e}}$ are the value of electron density and electron pressure deduced by comparing the theoretical and observed line ratios.

The SUMER sequence allows observation of the $2 \mathrm{p}^{2}{ }^{3} \mathrm{P}_{1}-2 \mathrm{~s} 2 \mathrm{p}{ }^{3} \mathrm{P}_{2}$ line at $762.00 \AA$. The $I(762.0 \AA) / I(629.7 \AA)$ ratio gives an estimate of the electron density at $\log T_{\mathrm{e}}(\mathrm{K})$ $\sim 5.40$.

The second line ratio involves two Si VII lines observed by EIS and belonging to the O-like isoelectronic sequence. They are the $2 \mathrm{~s}^{2} 2 \mathrm{p}^{4}{ }^{3} \mathrm{P}_{2}-2 \mathrm{~s} 2 \mathrm{p}^{5}{ }^{3} \mathrm{P}_{2}$ at $275.35 \AA$ and the $2 \mathrm{~s}^{2} 2 \mathrm{p}^{4}{ }^{3} \mathrm{P}_{1}-2 \mathrm{~s} 2 \mathrm{p}^{5}{ }^{3} \mathrm{P}_{1}$ at $275.67 \AA$. The $2 \mathrm{~s}^{2} 2 \mathrm{p}^{4}{ }^{3} \mathrm{P}-2 \mathrm{~s} 2 \mathrm{p}^{5}{ }^{3} \mathrm{P}$ transitions give rise to six closely spaced lines that, for higher ionization stages such as $\mathrm{Si}^{6+}$, show some relative density sensitivity. The $I(275.3 \AA) / I(275.7 \AA)$ ratio allows one to estimate the electron density at $\log T_{\mathrm{e}}(\mathrm{K}) \sim 5.75$.

The third ratio comes from a $\mathrm{P}$-like $\mathrm{Fe}$ line ratio. The circumstances are similar to the $\mathrm{O} v$ case. The term $3 \mathrm{~s}^{2} 3 \mathrm{p}^{3}{ }^{2} \mathrm{D}$ of Fe XII is metastable, and therefore lines that involve this term are density sensitive. The transitions between the ${ }^{2} \mathrm{D}$ and ${ }^{2} \mathrm{~F}$ terms produce three lines. EIS allows observation of the line $186.88 \AA$, which is a self-blend of the $3 s^{2} 3 p^{3}{ }^{3} D_{5 / 2}-3 s^{2} 3 p^{2} 3 d^{2} F_{7 / 2}$ transition with the $3 s^{2} 3 p^{3}{ }^{3} D_{3 / 2}-3 s^{2} 3 p^{2} 3 d{ }^{2} F_{5 / 2}$ transition. ${ }^{7}$ The other line is a strong resonance line at $193.51 \AA$ between the ground state and the $3 \mathrm{~s}^{2} 3 \mathrm{p}^{2} 3 \mathrm{~d}{ }^{4} \mathrm{P}_{3 / 2}$ level. The $I(193.5 \AA) / I(186.9 \AA)$ ratio provides a good density diagnostic at $\log T_{\mathrm{e}}(\mathrm{K}) \sim 6.10$.

The electron density of emitting plasma is evaluated by comparing the observed ratios with the theoretical values calculated within ADAS.

The three density-sensitive line ratios are listed in Table 5. This table reports the temperatures of line formation, which have been defined as the temperatures where the contribution functions peak, and the derived density and pressure.

The electron densities, which have been inferred from the comparison of the measurements with the theoretical ratios, would lead to the conclusion that the plasma is closer to having constant $P_{\mathrm{e}}$ rather than constant $N_{\mathrm{e}}$. The most likely pressure indicated by Table 5 is around $10^{15} \mathrm{~cm}^{-3} \mathrm{~K}$. However, due to the non-negligible scatter especially in the O v $I(762.0 \AA) / I$ $(629.7 \AA)$ and Si vII $I(257.3 \AA) / I(275.7 \AA)$ ratios, a larger grid of uniform $P_{\mathrm{e}}$ will be considered for the evaluation of the $G$ functions, using the following three values: $P_{\mathrm{e}}^{(1)} \simeq 5 \times 10^{14}$, $P_{\mathrm{e}}^{(2)} \simeq 1 \times 10^{15}$, and $P_{\mathrm{e}}^{(3)} \simeq 4 \times 10^{15} \mathrm{~cm}^{-3} \mathrm{~K}$. Additionally, in order to investigate the consequences of assuming a constant electron density, a constant $N_{\mathrm{e}}$ approximation for evaluation of the contribution functions will be used, by setting the electron density at a value equal to $10^{10} \mathrm{~cm}^{-3}$.

\footnotetext{
The other two lines of the $\mathrm{O}^{3+}$ ion may contribute to the blend. However, the DEM prediction shows that they contribute less than $0.01 \%$ to the blend So, they can be safely neglected.
}

\subsubsection{Si II, C II, and C III Intensities}

A general problem related to the solar models of the upper chromosphere/lower transition region arises from the uncertain structure of these atmospheric layers. The present observations include five $\mathrm{Si}$ II lines, which sample progressively higher layers of the upper chromosphere reaching the lower transition region. Therefore, they provide useful constraints on these atmospheric layers and permit deduction of information on the behavior of other lines formed in these regions, such as $\mathrm{He}_{\mathrm{I}}$ and He II lines. The analysis of the Si II resonance multiplets, performed by Lanzafame (1994), led to the conclusion that the lines belonging to the $3 \mathrm{~s}^{2} 3 \mathrm{p}^{2} \mathrm{P}-3 \mathrm{~s} 3 \mathrm{p}^{2}{ }^{2} \mathrm{~S}$ and $3 s^{2} 3 p^{2} \mathrm{P}-3 s^{2} 3 d^{2} \mathrm{D}$ multiplets are moderately optically thick. This affects the observed intensities, which may result in lower values than the intensities in the pure optically thin case. Since an appropriate DEM analysis requires that the lines involved in the integral inversion are strictly optically thin, these $\mathrm{Si}$ II line intensities have to be adjusted to be consistent with an optically thin study.

In addition, several lines of $\mathrm{C}$ II and $\mathrm{C}$ III are observed. The multiplets to which they belong have been extensively examined by Brooks et al. (2000), and the spectral lines emitted by these two carbon ions have been classified according to how much they are affected by opacity. The work of Brooks et al. (2000) has been taken as a point of reference to derive the correction factors for the silicon line intensities and as a source for the intensity corrections applied to the $\mathrm{C}_{\mathrm{II}}$ and $\mathrm{C}_{\text {III }}$ lines included in the present observations. They analyzed the emergent fluxes using an escape probability model to deduce the optical thickness of the considered lines. This technique is a relatively simple approach because it allows one to avoid the need to solve the full set of radiative transfer equations. It adopts the basic idea, established by Jordan (1967) and implemented by Brooks et al. (2000) and Fischbacher et al. (2000), of extracting optical depths from observed intensity ratios of lines arising from a common upper level, together with the escape probability model introduced by Holstein (1947) and McWhirter et al. (1965). According to this approach, the effect of opacity can be described with a correction factor applied to the radiative transition probability $A_{j \rightarrow i}$, so that $A_{j \rightarrow i}^{\text {thick }}=A_{j \rightarrow i}^{\text {thin }} g\left(\tau_{0}\right)$, where $g\left(\tau_{0}\right)$ is called the escape factor and $\tau_{0}$ is the mean optical depth at the central frequency of the line. In terms of intensity,

$$
I_{j \rightarrow i}^{\text {thick }}=g\left(\tau_{0}\right) I_{j \rightarrow i}^{\text {thin }}
$$

assuming that $g\left(\tau_{0}\right)$ depends only on the optical depth at the center of the line. The purpose of such an approach is to derive suitable correction factors that account for the photon absorption along the line of sight and use them to adjust the observed intensity for those lines that need to be included in the DEM analysis. Calling $I^{\text {obs }}$ the observed intensity of a line and $I^{\text {corr }}$ the intensity of the same line in an optically thin regime, from Equation (2) one obtains

$$
I^{\mathrm{corr}}=\frac{I^{\mathrm{obs}}}{g\left(\tau_{0}\right)} .
$$

Table 6 lists the lines to which the optically thick correction has been applied and the corresponding enhanced intensity indicated by $I^{\text {corr }}$. The note $(a)$ indicates the escape factors taken from Brooks et al. (2000). For the silicon lines the escape 
Table 6

Intensity Corrected Using the Escape Factor $g\left(\tau_{0}\right)$ for Those Lines That Are Affected by Moderate Opacity

\begin{tabular}{|c|c|c|c|c|}
\hline Line & & $g\left(\tau_{0}\right)$ & $I^{\mathrm{obs}}$ & $I^{\text {corr }}$ \\
\hline$b \mathrm{C}_{\mathrm{III}}$ & $538.20 \AA$ & $0.99^{(a)}$ & 12.6 & 12.8 \\
\hline $\mathrm{C}_{\mathrm{III}}$ & $1174.93 \AA$ & $0.93^{(a)}$ & 43.1 & 46.4 \\
\hline $\mathrm{C}_{\text {III }}$ & $1175.26 \AA$ & $0.94^{(a)}$ & 36.4 & 38.7 \\
\hline $\mathrm{C}_{\text {III }}$ & $1176.37 \AA$ & $0.93^{(a)}$ & 40.7 & 43.8 \\
\hline Si II & $1190.42 \AA$ & 0.61 & 19.9 & 32.6 \\
\hline Si II & $1264.74 \AA$ & 0.27 & 119.7 & 440.4 \\
\hline Si II & $1265.00 \AA$ & 0.95 & 48.7 & 51.3 \\
\hline $\mathrm{Si}$ II & $1304.37 \AA$ & 0.77 & 52.1 & 67.5 \\
\hline $\mathrm{Si}$ II & $1309.28 \AA$ & 0.64 & 85.0 & 131.9 \\
\hline $\mathrm{C}_{\text {II }}$ & $1334.53 \AA$ & $0.73^{(a)}$ & 813.7 & 1114.7 \\
\hline $\mathrm{C}_{\text {II }}$ & $1335.66 \AA$ & $0.84^{(a)}$ & 61.7 & 73.5 \\
\hline $\mathrm{C}_{\text {II }}$ & $1335.71 \AA$ & $0.64^{(a)}$ & 1021.8 & 1597.2 \\
\hline
\end{tabular}

Note. The intensity units are $\operatorname{erg} \mathrm{cm}^{-2} \mathrm{~s}^{-1} \mathrm{sr}^{-1}$. For the prefix $b$ see Table 2 .

factors have been calculated using the same procedure as Brooks et al. (2000). Although the correction factors can be large for these lines, $g\left(\tau_{0}\right)$ is not a free parameter and is strongly constrained by the evaluation methodology. In this treatment, it has been assumed that all the observed Si II lines originate from the same atmospheric layer. However, as pointed out by Lanzafame (1994), the region of formation of the $\mathrm{Si}$ II multiplets embraces regions with different depth of formation. This can affect the results obtained for the two observed components of the Si II $1265 \AA$ multiplet and the line at $1190.42 \AA$. For this reason, the strongest component of the $3 s^{2} 3 p{ }^{2} \mathrm{P}-3 s 3 p^{2}{ }^{2} \mathrm{~S}$ multiplet has been selected to perform the integral inversion, while the other $\mathrm{Si}$ II lines have been used in the forward sense.

\subsubsection{Elemental Abundances}

The abundance analysis is done in two steps. First, known values are used. The photospheric and coronal abundance collection of Phillips et al. (2008) is adopted as the main reference. Additionally, coronal abundances from three different models have been investigated: low-FIP enhancement (e.g., elements with their first ionisation potential, FIP, lower than $10 \mathrm{eV}$ are enhanced with respect to their photospheric values) from Feldman \& Widing (2003), high-FIP depletion (e.g., elements with their FIP greater than $10 \mathrm{eV}$ are depleted with respect to their photospheric values) from Meyer (1985), and hybrid (e.g., a combination of low-FIP enhancement and highFIP depletion) from Fludra \& Schmelz (1999).

Second, once the observed lines to perform the integral inversion have been selected and the electron density/pressure has been estimated, the relative values of elemental abundances are adjusted to minimize deviation from observations. In order to deduce relative elemental abundances from the present observations, two elements have been selected as a starting point: oxygen, which is a high-FIP element, and silicon, which is a low-FIP element. For the available ions of each of these two elements, lines that do not show evident blends have been selected. A preliminary DEM analysis has been performed independently for oxygen and silicon. This is necessary to check whether the relative intensities of the ions of oxygen and silicon are well calibrated and their theoretical reconstruction is

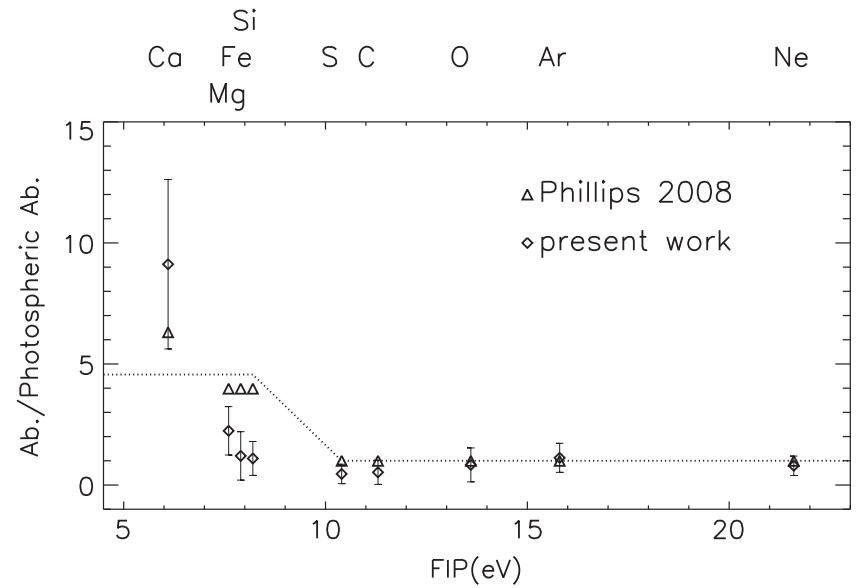

Figure 2. Elemental abundances for the SUMER-CDS-EIS joint observations of 2009 April compared with the Phillips et al. (2008) coronal abundances. Abundances are plotted as ratios with the photospheric values taken from the collection of Phillips et al. (2008).

reliable within the observational uncertainties, without involving abundance values. Then, oxygen and silicon ions have been analyzed together, by means of DEM. In evaluating their abundances, it has been assumed that the oxygen abundances (with respect to hydrogen) in the transition region and corona are the same as in the photosphere. The initial value has been taken from the photospheric abundances of Phillips et al. (2008). The silicon abundance has been adjusted in order to give the minimum deviation from observations. Once the relative abundance between oxygen and silicon has been estimated, the available neon ions have been added to the analysis. Following the suggestion of Feldman \& Widing (2007), the neon abundance has been assumed to be $\sim 0.15$ of the oxygen abundance. Thus, a decrease in the oxygen abundance implies a similar decrease in the neon abundance. Once this preliminary analysis has been done, the abundances for the other elements $(\mathrm{C}, \mathrm{S}, \mathrm{Fe}, \mathrm{Mg}, \mathrm{Ar}, \mathrm{Ca})$ included in the present work are evaluated using the approach described by Lanzafame et al. (2005). For helium, the abundance from Meyer (1985) has been adopted. Figure 2 shows the set of abundances evaluated for this work together with the Phillips et al. (2008) coronal abundances for comparison. The abundances are shown as ratios to their photospheric values, taken from Phillips et al. (2008). Interesting results are found for $\mathrm{Si}$ and $\mathrm{Fe}$ abundances. Their values are close to photospheric values. Similar results have been found by Lanzafame et al. (2002) in the analysis of data of an active region observed by SERTS-89. Here, the same behavior of $\mathrm{Si}$ and $\mathrm{Fe}$ abundances is found for the quiet Sun as well.

\subsubsection{Comparison between Observed and Predicted Intensities}

Table 7 shows the lines used for the DEM inversion, while Table 8 includes all the other lines. $I^{\text {obs }}$ and err $^{\text {obs }}$ are the observed intensities and the respective uncertainties (fit+ calibration). $T_{\mathrm{e}}$ is the electron temperature of the peak of the line contribution functions. Reconstructed intensites are given in four cases where the $G\left(T_{\mathrm{e}}\right)$ functions are computed in the uniform pressure approximation, with $P_{\mathrm{e}}^{(1)}=5 \times 10^{14}$, $P_{\mathrm{e}}^{(2)}=1 \times 10^{15}$, and $P_{\mathrm{e}}^{(3)}=4 \times 10^{15} \mathrm{~cm}^{-3} \mathrm{~K}$, and uniform density approximation, with $N_{\mathrm{e}}=10^{10} \mathrm{~cm}^{-3}$. The ratios between observed and reconstructed intensities are reported 
Table 7

Lines Used for Integral Inversion

\begin{tabular}{lcrrrrrr}
\hline \hline Line & $\log \left(T_{\mathrm{e}}\right)$ & \multicolumn{1}{c}{$I^{\text {obs }}$} & err $^{\text {obs }}$ & $R_{1}$ & $R_{2}$ & $R_{3}$ & $R_{4}$ \\
\hline Si II $1309.28^{\mathrm{N}}$ & 4.30 & 131.9 & 42.2 & 1.0 & 1.0 & 1.0 & 1.0 \\
C III $1174.93^{\mathrm{N}}$ & 4.80 & 46.4 & 15.6 & 1.0 & 1.0 & 1.0 & 1.0 \\
O III $599.60^{\mathrm{N}}$ & 4.95 & 42.7 & 13.9 & 1.0 & 1.0 & 1.0 & 1.0 \\
Ne IV $543.89^{\mathrm{E}}$ & 5.15 & 8.1 & 2.7 & 1.0 & 1.0 & 1.0 & 1.0 \\
O IV $787.71^{\mathrm{N}}$ & 5.20 & 75.4 & 29.5 & 0.8 & 0.8 & 0.8 & 0.8 \\
O v $629.73^{\mathrm{N}}$ & 5.30 & 458.5 & 128.3 & 1.0 & 1.0 & 1.0 & 1.0 \\
O VI $184.12^{\mathrm{N}}$ & 5.45 & 8.1 & 2.3 & 1.0 & 1.0 & 1.0 & 0.9 \\
Fe VIII $186.60^{\mathrm{N}}$ & 5.70 & 21.7 & 5.2 & 0.9 & 0.9 & 1.0 & 1.0 \\
Si VII $275.67^{\mathrm{N}}$ & 5.75 & 2.8 & 0.9 & 1.0 & 1.0 & 1.0 & 1.0 \\
Fe XII $193.51^{\mathrm{N}}$ & 6.10 & 43.3 & 9.5 & 1.0 & 1.0 & 1.0 & 1.0 \\
Si XII $520.66^{\mathrm{E}}$ & 6.25 & 3.7 & 1.3 & 1.0 & 1.0 & 1.0 & 1.0 \\
\hline
\end{tabular}

Note. Intensities are in erg cm $\mathrm{s}^{-2} \mathrm{sr}^{-1}$. $\mathrm{N}$ is for NIST; E is for (Edlén $(1983,1985))$. The wavelengths used are the preferred laboratory wavelengths in $\AA$. The ratios are the observed to DEM reconstructed intensities: $R_{1}=I^{\text {obs }} / I^{\text {rec }}$ $\left(P_{\mathrm{e}}^{(1)}\right), R_{2}=I^{\mathrm{obs}} / I^{\mathrm{rec}}\left(P_{\mathrm{e}}^{(2)}\right), R_{3}=I^{\mathrm{obs}} / I^{\mathrm{rec}}\left(P_{\mathrm{e}}^{(3)}\right)$, and $R_{4}=I^{\mathrm{obs}} / I^{\mathrm{rec}}\left(N_{\mathrm{e}}\right)$.

in Tables 7 and 8. Figure 3 shows the four DEM curves as a function of electron temperature, using the set of elemental abundances defined in Section 4.1.3. The difference of the DEM derived from the three constant $P_{\mathrm{e}}$ approximations and the constant $N_{\mathrm{e}}$ model is within $15 \%$ except for the temperature ranges $\log T_{\mathrm{e}}=4.40-4.60$ and $\log T_{\mathrm{e}}=5.90-6.10$, where the model using $P_{\mathrm{e}}^{(3)}$ and $P_{\mathrm{e}}^{(2)}$ departs from the uniform $N_{\mathrm{e}}$ approximation between $30 \%$ and $40 \%$. As shown in Table 8 and Figure 4(B), most of the lines are predicted within the observational uncertainties. However, an inconsistency is found in the reconstructed intensity of the Si XI $303.33 \AA$ line (Figure 4(A)), which is observed in the second order of CDS. For this line, $I^{\mathrm{obs}} / I^{\text {rec }} \sim 9-12$. This discrepancy is consistent with Lanzafame et al. (2002, 2005) spectral analysis, where $I^{\text {obs }} / I^{\text {rec }} \sim 10$. Possible blends were investigated by Lanzafame et al. (2005), but none of those considered contribute appreciably to the observed intensity. Moreover, this Si XI line may suffer from radiative pumping due to the close vicinity to the strong He II $303.78 \AA$ doublet. Another cause may be sought in the CDS calibration at the second order, after SOHO's loss in 1998. Obviously, this can affect also the He II line. A significant uncertainty in the second order of the CDS calibration (e.g., by a factor $\sim 5$ ) would explain partially the Si XI $303.33 \AA$ discrepancy and would reduce the enhancement of $\mathrm{He}$ II $303.78 \AA$ to a factor comparable with $\mathrm{He}$ II $256.32 \AA$. However, a further investigation of CDS calibration at the second order needs dedicated observations and analyses. Further work will be planned to examine this behavior.

\subsubsection{Radiative Transfer Calculations}

In order to include consistently the effect of opacity and to compare the predicted intensities with the optically thin reconstruction for helium lines, non-LTE radiative transfer calculations have been performed using MULTI. First, semiempirical models of the Sun's atmosphere have been built up by combining the photospheric and chromospheric part of the VALC model (Vernazza et al. 1981) with the transition region part derived from the DEM calculated in this work. The DEM was integrated to obtain $P_{\mathrm{e}}$ as a function of $T_{\mathrm{e}}$ in the transition region and corona, taking $\log N_{\mathrm{e}}=9.4$ at $\log T_{\mathrm{e}}=5.75(\mathrm{see}$ Table 5) as the boundary condition. The total pressure was then obtained from $P_{\mathrm{e}}$, assuming the ratio of neutral to ionized hydrogen as a function of $T_{\mathrm{e}}$ given in the VALC model. The column mass was therefore derived from the hydrostatic equilibrium equation. Turbulent pressure was included following Jordan (1992). Note that this procedure is very similar to that adopted by Pietarila \& Judge (2004), the main difference being that the DEM has been integrated instead of the EM. Between $\log T(\mathrm{~K}) \approx 3.8$ (chromosphere) and 4.3 the DEM is constrained by the $\mathrm{Si}$ II $1309.28 \AA$ line and the extrapolation to lower temperature adopted, which makes the resulting semiempirical atmospheric model somewhat uncertain. To overcome this limitation, we built a grid of semi-empirical models by joining the transition region model below $\log T_{\mathrm{e}}=4.3$ to the chromospheric temperature plateau with different temperature gradients. The model that best reproduced the $\mathrm{Si}$ II lines was then used to estimate the $\mathrm{He}$ I lines.

The results of the radiative transfer calculations for $\mathrm{He}$ I are shown in Table 9 for the intercombination line and the first two resonance lines (584.33 and $537.03 \AA$ ).

Additionally, the impact of the new He I atomic data on the line reconstruction has been estimated by running the radiative transfer code with the new atomic data using the Pietarila \& Judge (2004) atmospheric model. For the He I $584.33 \AA$ line an intensity of a factor of 2 higher than computed by Pietarila \& Judge (2004) has been obtained, while the He I $537.03 \AA$ intensity is approximately the same.

\subsection{Results}

Table 10 shows the enhancement factors derived using the DEM analysis. All the EUV $\mathrm{He}_{\mathrm{I}}$ and $\mathrm{He}$ II resonance lines observed by CDS and EIS have been included in this study. Also, the first line shown in Table 10 is the intercombination line of $\mathrm{He}_{\mathrm{I}}$ observed by $\mathrm{CDS}$ at $591.41 \AA$. Because of its optically thin behavior, a proper DEM analysis should give reliable results for its predicted intensity. It should be noted that it is a very weak line, with low integrated intensity, hence the uncertainties related to the fit and the calibration procedures are quite high compared to the observed flux. However, the reconstructed intensity for this line is within the observational uncertainties, suggesting that the main mechanism that affects the resonance lines of neutral helium in these observations may be the opacity. The comparison between observed and predicted intensities for the $\mathrm{He}$ I resonance lines does not show an enhancement greater than a factor of 2, which is the lower limit in Pietarila \& Judge (2004) calculations. Furthermore, the first line of the resonance series shows a depletion of a factor of $\sim 2$, again supporting the opacity mechanisms. This has been also confirmed by non-LTE radiative transfer calculations (Section 4.1.5), where the $\mathrm{He}$ I reconstructed intensities are in good agreement with observations, with some tendency to underestimate the weaker line. In particular, a grid of atmospheric models has been tested in order to give a much stronger constraint on the region of $\mathrm{He}$ I formation, and a direct comparison with the Pietarila \& Judge (2004) model has also been done.

Table 11 allows a comparison of the present enhancement factors and the ones derived by Jordan (1975), Macpherson \& Jordan (1999), and Pietarila \& Judge (2004). Differences with ealier calculations for neutral helium arise mostly from the new revised atomic data and modeling employed not only for helium but also for the other ions used in deriving the DEM curve. In addition, two other main aspects contribute to this difference. First, the atmospheric model for non-LTE 
Table 8

Lines Intensities Predicted by the DEM Illustrated in Figure 3

\begin{tabular}{|c|c|c|c|c|c|c|c|}
\hline Line & $\log \left(T_{\mathrm{e}}\right)$ & $I^{\mathrm{obs}}$ & err $^{\text {obs }}$ & $R_{1}$ & $R_{2}$ & $R_{3}$ & $R_{4}$ \\
\hline \multicolumn{8}{|l|}{ SUMER } \\
\hline $\mathrm{C}_{\text {II }^{\mathrm{N}}} 1335.71$ & 4.45 & 1596.7 & 523.7 & 1.0 & 0.6 & 0.9 & 0.8 \\
\hline $\mathrm{C}_{\text {II }^{\mathrm{N}}} 1334.53$ & 4.45 & 1114.7 & 367.9 & 0.9 & 0.8 & 1.1 & 1.0 \\
\hline $\mathrm{Si}_{\text {II }}^{\mathrm{N}} 1304.37$ & 4.30 & 67.5 & 24.0 & 1.6 & 1.0 & 1.9 & 1.8 \\
\hline $\mathrm{Si}_{\text {II }}^{\mathrm{N}} 1265.00$ & 4.30 & 51.3 & 17.3 & 1.4 & 0.9 & 1.7 & 1.5 \\
\hline $\mathrm{Si}_{\text {II }}^{\mathrm{N}} 1264.74$ & 4.30 & 440.5 & 142.3 & 1.4 & 0.9 & 1.7 & 1.5 \\
\hline $\mathrm{Si}_{\mathrm{II}}^{\mathrm{N}} 1190.42$ & 4.30 & 32.6 & 10.5 & 1.1 & 0.7 & 1.3 & 1.2 \\
\hline $\mathrm{S}_{\text {III }}^{\mathrm{N}} 1190.17$ & 4.75 & 2.0 & 0.8 & 0.8 & 1.0 & 1.1 & 0.9 \\
\hline $\mathrm{C}$ III $^{\mathrm{N}} 1176.37$ & 4.80 & 43.8 & 8.4 & 0.9 & 0.9 & 0.9 & 0.9 \\
\hline $\mathrm{C}$ IIII $^{\mathrm{N}} 1175.26$ & 4.80 & 38.7 & 13.2 & 1.0 & 1.0 & 1.0 & 1.0 \\
\hline $\mathrm{S}$ III $^{\mathrm{N}} 1077.14$ & 4.80 & 2.8 & 0.9 & 0.9 & 0.9 & 1.0 & 0.9 \\
\hline$b \mathrm{O} \mathrm{IV}{ }^{\mathrm{N}} 790.19+790.11$ & 5.20 & 179.7 & 45.9 & 1.0 & 1.0 & 1.0 & 1.0 \\
\hline $\mathrm{Na} \mathrm{VIII}^{\mathrm{N}} 789.78$ & 5.85 & 2.1 & 1.9 & 1.0 & 0.9 & 0.8 & 0.7 \\
\hline $\mathrm{Ne}_{\mathrm{VIII}}^{\mathrm{N}} 780.30$ & 5.75 & 42.4 & 13.3 & 0.8 & 0.8 & 0.7 & 0.7 \\
\hline $\mathrm{Ne}_{\text {VIII }}^{\mathrm{N}} 770.42$ & 5.75 & 96.7 & 30.7 & 0.9 & 0.9 & 0.8 & 0.8 \\
\hline Mg VIII ${ }^{\mathrm{N}} 762.65$ & 5.90 & 0.3 & 0.1 & 1.0 & 1.0 & 1.0 & 0.8 \\
\hline $\mathrm{O} \mathrm{v}^{\mathrm{N}} 761.99$ & 5.30 & 8.8 & 2.9 & 1.1 & 1.0 & 0.8 & 0.9 \\
\hline $\mathrm{O} \mathrm{v}^{\mathrm{N}} 761.13$ & 5.30 & 0.7 & 0.3 & 1.0 & 0.7 & 0.2 & 0.4 \\
\hline $\mathrm{He} \mathrm{I}^{\mathrm{N}} 584.33$ & 4.45 & 574.1 & 177.4 & 0.5 & 0.5 & 0.7 & 0.5 \\
\hline \multicolumn{8}{|l|}{ CDS } \\
\hline $\mathrm{O}_{\text {III }}^{\mathrm{N}} 597.82$ & 4.95 & 2.8 & 1.2 & 1.1 & 1.1 & 1.1 & 1.2 \\
\hline $\mathrm{He}_{\mathrm{I}}^{\mathrm{N}} 591.41$ & 4.45 & 0.16 & 0.08 & 1.6 & 1.7 & 2.2 & 1.6 \\
\hline $\operatorname{Ar}{ }^{N}{ }^{N} 585.75$ & 5.50 & 5.3 & 3.7 & 0.9 & 0.9 & 1.0 & 1.1 \\
\hline $\mathrm{He}^{\mathrm{N}} 584.33$ & 4.45 & 638.8 & 203.9 & 0.5 & 0.6 & 0.7 & 0.5 \\
\hline $\mathrm{Ca} \mathrm{X}^{\mathrm{E}} 574.01$ & 5.80 & 8.6 & 2.8 & 1.0 & 0.9 & 0.8 & 0.7 \\
\hline $\mathrm{O}_{\mathrm{IV}}^{\mathrm{N}} 555.76$ & 5.20 & 30.18 & 9.8 & 0.9 & 0.9 & 1.0 & 0.9 \\
\hline $\mathrm{O} \mathrm{IV}^{\mathrm{N}} 554.51$ & 5.20 & 135.69 & 43.0 & 0.9 & 0.9 & 0.9 & 0.9 \\
\hline $\mathrm{O}_{\mathrm{IV}}{ }^{\mathrm{N}} 554.08$ & 5.20 & 56.03 & 18.8 & 0.9 & 0.9 & 0.9 & 0.9 \\
\hline $\mathrm{O} \mathrm{IV}^{\mathrm{N}} 553.33$ & 5.20 & 30.3 & 9.7 & 1.0 & 1.0 & 1.0 & 1.0 \\
\hline $\mathrm{Ne} \operatorname{IV}^{\mathrm{E}} 542.07$ & 5.15 & 5.3 & 1.8 & 1.0 & 1.0 & 1.0 & 1.0 \\
\hline$b \mathrm{C} \mathrm{III}^{\mathrm{N}}+\mathrm{O}$ II $^{\mathrm{N}} 538.20$ & 4.80 & 12.8 & 1.0 & 1.0 & 1.0 & 1.1 & 0.9 \\
\hline $\mathrm{He}^{\mathrm{N}} 537.03$ & 4.45 & 71.8 & 22.9 & 1.7 & 1.8 & 2.2 & 1.7 \\
\hline $\mathrm{O}_{\text {III }}^{\mathrm{N}} 525.80$ & 4.95 & 23.7 & 7.5 & 1.3 & 1.3 & 1.3 & 1.4 \\
\hline $\mathrm{He}^{\mathrm{N}} 522.21$ & 4.45 & 24.9 & 8.0 & 1.7 & 1.7 & 1.7 & 1.9 \\
\hline $\mathrm{He}^{\mathrm{N}} 515.62$ & 4.45 & 13.6 & 4.3 & 0.9 & 0.9 & 1.0 & 1.0 \\
\hline $\mathrm{He}_{\text {II }}{ }^{\mathrm{N}} 303.78$ & 4.90 & 5383.7 & 1717.6 & 13.2 & 12.9 & 12.6 & 13.3 \\
\hline $\mathrm{Si} \mathrm{XI}^{\mathrm{E}} 303.33$ & 6.15 & 395.7 & 205.7 & 11.6 & 11.2 & 10.3 & 9.3 \\
\hline \multicolumn{8}{|l|}{ EIS } \\
\hline $\mathrm{O}_{\mathrm{IV}^{\mathrm{N}}}^{\mathrm{N}} 279.93$ & 5.20 & 3.5 & 1.3 & 0.8 & 0.8 & 0.9 & 0.8 \\
\hline $\mathrm{O} \mathrm{IV}^{\mathrm{N}} 279.63$ & 5.20 & 2.0 & 0.8 & 0.9 & 0.9 & 1.0 & 0.9 \\
\hline $\mathrm{Si} \mathrm{VII}^{\mathrm{N}} 275.35$ & 5.75 & 16.2 & 3.8 & 0.9 & 1.0 & 1.1 & 1.1 \\
\hline$b \mathrm{He}$ II $^{\mathrm{N}}+\mathrm{Si} \mathrm{x}^{\mathrm{N}}+b \mathrm{Fe} \mathrm{x}^{\mathrm{N}}+\mathrm{Fe} \mathrm{xIII}^{\mathrm{N}} 256.32$ & 4.90 & 222.8 & 47.4 & 5.8 & 5.7 & 5.4 & 5.3 \\
\hline$b \mathrm{Fe} \mathrm{XII}^{\mathrm{N}}\left(+\mathrm{O} \mathrm{IV}^{\mathrm{N}}\right) 186.88$ & 6.10 & 13.6 & 3.4 & 1.3 & 0.9 & 0.5 & 0.3 \\
\hline $\mathrm{O} \mathrm{vI}^{\mathrm{N}} 183.94$ & 5.45 & 4.3 & 1.5 & 1.1 & 1.1 & 1.0 & 1.0 \\
\hline
\end{tabular}

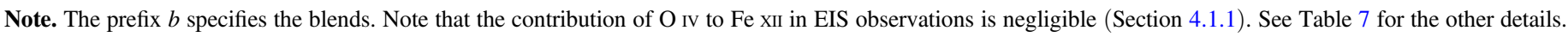

calculations in Section 4.1.5 is derived by the DEM, which is a stronger constraint for the temperature gradient than the EM adopted by Pietarila \& Judge (2004). Second, the model is further constrained between $3.8<\log T<4.3$ using the $\mathrm{Si}$ II lines, which has not been done by Pietarila \& Judge (2004).

Nevertheless, this analysis examines a quiet-Sun region near the disk center only. As a consequence, any information on helium behavior in active regions or coronal holes is not provided here. Further work will extend this analysis to a wider range of solar plasma conditions, in order to give more constraints on the new enhancement factors obtained for $\mathrm{He}$ I.
By contrast, the computed enhancement for He II agrees with that of Jordan (1975) and Macpherson \& Jordan (1999). As suggested by Macpherson \& Jordan (1999), a full non-LTE radiative transfer calculations may give more reliable constraints to the He II enhancement factor, without including ad hoc assumptions on the fractions of escaping photons. However, other issues may affect the intensities of both Не г 303.78 and $\mathrm{He}$ II $256.32 \AA$ lines, ranging from calibration uncertainty to line blending. Moreover, even if further unknown blends might explain enhancement of a factor of 5-6 of the He II $256.32 \AA$ line, it is unlikely that instrument effects can explain the higher enhancement of $\mathrm{He}$ II $303.78 \AA$. 


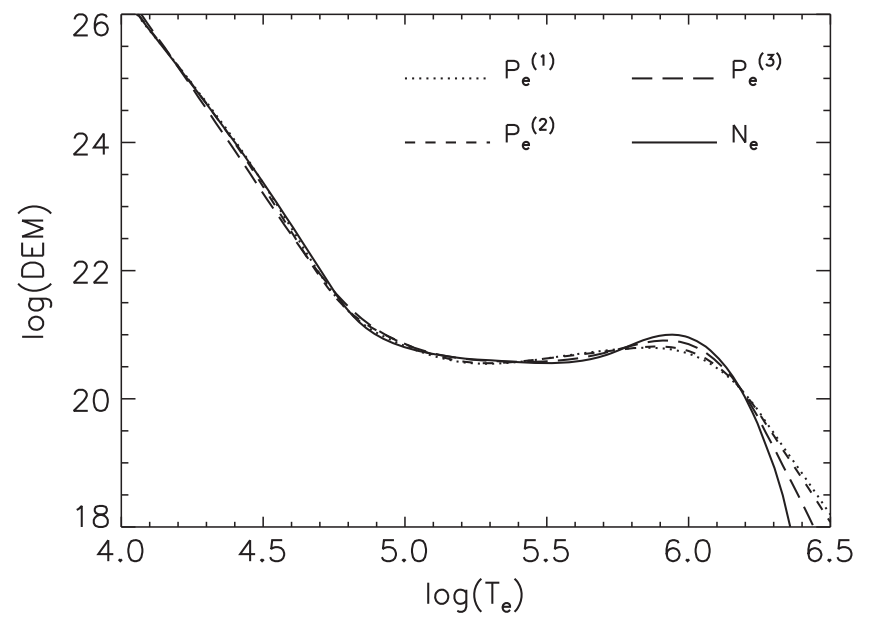

Figure 3. DEM for the averaged solar quiet region using EUV line intensities observed by SUMER, CDS, and EIS. The curves are derived assuming uniform $P_{\mathrm{e}}\left(P_{\mathrm{e}}^{(1)}=5 \times 10^{14}, P_{\mathrm{e}}^{(2)}=1 \times 10^{15}\right.$, and $\left.P_{\mathrm{e}}^{(3)}=4 \times 10^{15} \mathrm{~cm}^{-3} \mathrm{~K}\right)$ or $N_{\mathrm{e}}$ $\left(N_{\mathrm{e}}=10^{10} \mathrm{~cm}^{-3}\right)$ in the evaluation of contribution functions.

Assuming that the discrepancy for neutral helium is solved by a consistent analysis and the use of appropriate atomic data, the enhancement of He II may be explained by some processes that could selectively enhance ionized helium intensities. A suggestion may be sought in the relationship between the photospheric magnetic flux and the EUV emission (Fludra \& Ireland 2008; Fludra \& Warren 2010). The solar magnetic flux, generated in the convection zone, passes through the partially ionized photospheric and chromospheric plasma before it can appear high up in the upper transition region and solar corona. This connection between the lower and upper layers of the
Table 9

Comparison between the He I Line Intensities Reconstructed by Radiative Transfer Calculations $\left(I^{\mathrm{RT}}\right)$ and Observations $\left(I^{\mathrm{obs}}\right)$

\begin{tabular}{lcccc}
\hline \hline He I Line $(\AA)$ & $I^{\text {obs }}$ & err ${ }^{\text {obs }}$ & $I^{\mathrm{RT}}$ & $I^{\mathrm{RTPT}}$ \\
\hline 591.41 & 0.16 & 0.08 & 0.16 & $\ldots$ \\
$584.33 \mathrm{c}$ & 638.8 & 203.9 & 739.3 & 314.7 \\
$584.33 \mathrm{~s}$ & 574.1 & 177.4 & 739.3 & 314.7 \\
537.03 & 71.8 & 22.9 & 30.6 & 14.8 \\
\hline
\end{tabular}

Note. Also reported are the line intensities obtained using the Pietarila \& Judge (2004) atmospheric model $\left(I^{\mathrm{RTPT}}\right)$. The suffix c indicates the line observed by CDS, while s by SUMER.

\section{Table 10}

Ratios between Observed and Predicted Intensities for the Intercombination Line of $\mathrm{He} \mathrm{I}$ and the Resonance Lines of $\mathrm{He} \mathrm{I}$ and $\mathrm{He}$ II, Using Both Uniform Density $\left(\mathrm{R}\left(N_{\mathrm{e}}\right)\right)$ and Uniform Pressure $\left(R\left(P_{\mathrm{e}}^{(1)}\right), R\left(P_{\mathrm{e}}^{(2)}\right)\right.$, and $\left.R\left(P_{\mathrm{e}}^{(3)}\right)\right)$

\begin{tabular}{lrrrr}
\hline \hline Line $(\AA)$ & $R\left(P_{\mathrm{e}}^{(1)}\right)$ & $R\left(P_{\mathrm{e}}^{(2)}\right)$ & $R\left(P_{\mathrm{e}}^{(3)}\right)$ & $R\left(N_{\mathrm{e}}\right)$ \\
\hline He I & & & & \\
591.41 & $1.6 \pm 0.8$ & $1.7 \pm 0.9$ & $2.2 \pm 1.1$ & $1.6 \pm 0.9$ \\
515.62 & $0.9 \pm 0.3$ & $0.9 \pm 0.3$ & $1.0 \pm 0.3$ & $1.0 \pm 0.3$ \\
522.21 & $1.7 \pm 0.5$ & $1.7 \pm 0.5$ & $1.7 \pm 0.5$ & $1.9 \pm 0.6$ \\
537.03 & $1.7 \pm 0.5$ & $1.8 \pm 0.6$ & $2.2 \pm 0.7$ & $1.7 \pm 0.6$ \\
$584.33 c$ & $0.5 \pm 0.2$ & $0.6 \pm 0.2$ & $0.7 \pm 0.2$ & $0.5 \pm 0.2$ \\
$584.33 s$ & $0.5 \pm 0.1$ & $0.5 \pm 0.1$ & $0.7 \pm 0.2$ & $0.5 \pm 0.2$ \\
\hline He II & & & & \\
303.78 & $13.2 \pm 4.1$ & $12.9 \pm 4.0$ & $12.6 \pm 3.9$ & $13.3 \pm 4.0$ \\
256.32 & $5.8 \pm 1.3$ & $5.7 \pm 1.3$ & $5.4 \pm 1.3$ & $5.3 \pm 1.3$ \\
\hline
\end{tabular}

Note. For the suffixes c and s see Table 9.
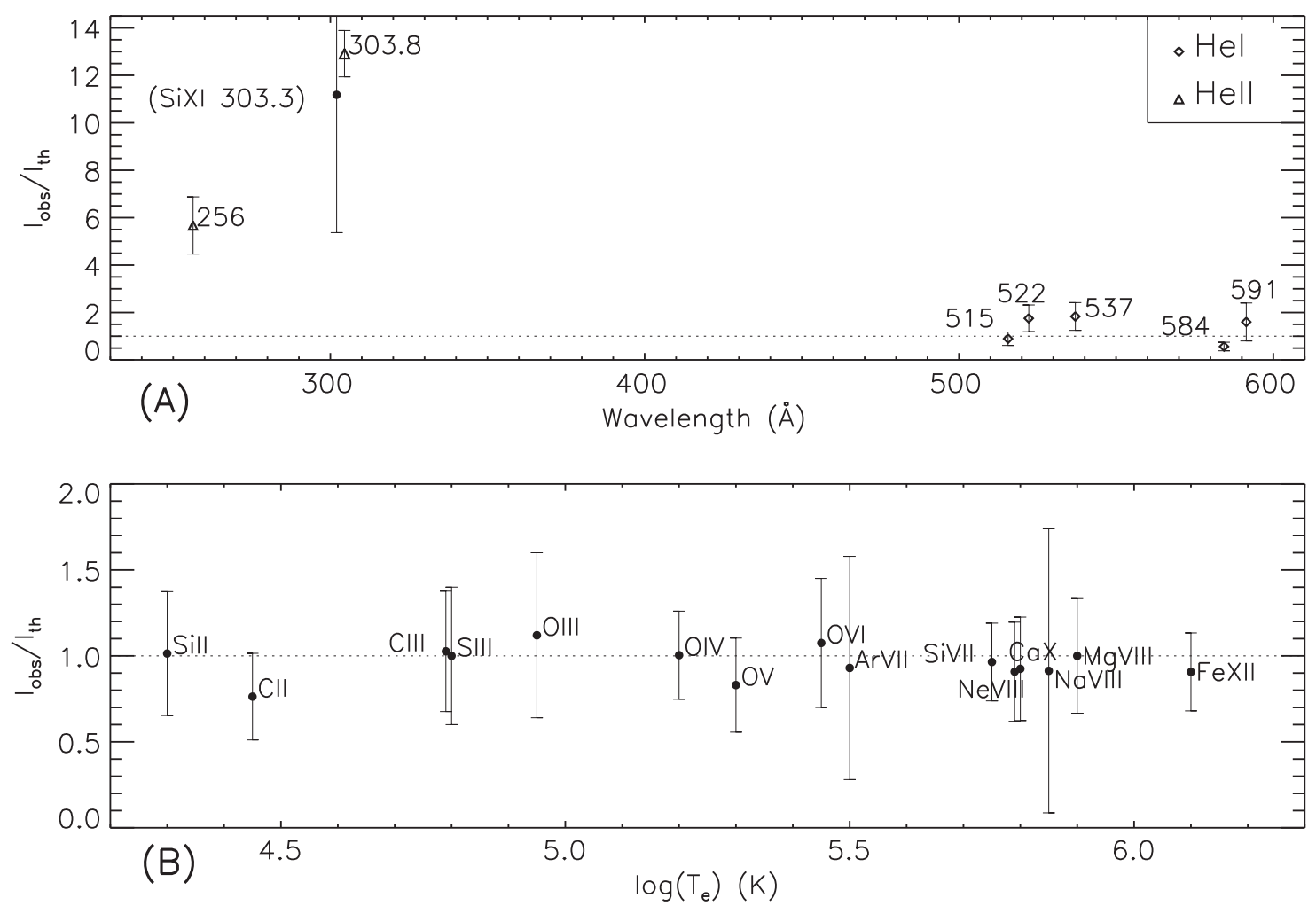

Figure 4. Ratios between the observed and predicted intensities for the helium lines + Si Xi $303.33 \AA$ as a function of wavelength $(a)$ and for the lines listed in Table 8 as a function of the electron temperature where the line contribution functions peak $(b)$. 
Table 11

Comparison between the Enhancement Factors Provided in the Literature and Those Derived by the Present Optically Thin and non-LTE Analysis

\begin{tabular}{lcc}
\hline \hline Source & He I & He II \\
\hline Jordan (1975) & 15 & 5.5 \\
Macpherson \& Jordan (1999) & $10-14$ & $13-25$ \\
Pietarila \& Judge (2004) & $2-5$ & $2-13$ \\
Present work & $0.5-2$ & $5-13$ \\
\hline
\end{tabular}

solar atmosphere might affect both the distribution of intensities and their integrated values of EUV transition region lines, providing different effects for neutral and ionized elements. Additionally, Judge (2008), following the suggestion of Judge \& Pietarila (2004), proposed a scenario that takes into account the spatial relationship among the observed chromosphere, transition region, and corona. He speculated that crossfield diffusion and a subsequent parallel conduction may affect line intensity values, giving an explanation of helium enhancements. The unexpected behavior of neutral helium lines, which show an enhancement close to one, while single ionized helium lines have a large enhancement, might suggest also that some other mechanism affects He II only. A recent investigation has been done by Golding et al. (2014). They analyzed the ionization timescales of helium in the chromosphere and transition region and found that He II $303.78 \AA$ is sensitive to non-equilibrium effects, which may affect substantially the observed intensity.

\section{CONCLUSIONS AND DISCUSSIONS}

The main objective of this work is to provide a complete and accurate approach to the investigation of the $70 \mathrm{yr}$ old problem of helium enhancement in the solar upper atmosphere, using state-of-the-art atomic data and multi-instrument observations.

The three EUV spectrometers, SOHO/SUMER, SOHO/CDS, and Hinode/EIS, employed in this project cover all together a large range of temperatures (from about $10^{4}$ to $10^{7} \mathrm{~K}$ ), allowing a comprehensive investigation of the solar atmosphere with the extension of the DEM analysis to temperature and density typical of the upper chromosphere.

In addition, this work is focused on the review and update of atomic data for light elements, concentrating on the precision and accuracy of the previous calculations and performing new computations to extend and top-up preferred data from the literature. A recommendation of atomic data with appropriate accuracy and including finite density effects is very important to avoid interpretation errors (Giunta et al. 2012). New calculations for silicon ionization, for both ground to ground state ionization and metastable resolved, have been performed and used for this analysis.

The combined use of new specific observations and revised atomic data has lead to the suggestion that the main mechanism that affects the intensity behavior of the resonance lines of neutral helium in these observations is the opacity. The comparison between observed and reconstructed intensities for He I resonance lines and the intercombination line does not show a real enhancement. By contrast, the first line of the $1 \mathrm{~s}^{2}{ }^{1} \mathrm{~S}-1 \mathrm{~s} n \mathrm{p}{ }^{1} \mathrm{P}$ resonance series shows a depletion of a factor of $\sim 2$, due to the opacity effect. Different conclusions have been found for single ionized helium lines. The enhancement factors obtained here agree with those of previous work. The different behavior of $\mathrm{He}_{\mathrm{I}}$ and $\mathrm{He}$ II lines may lead to the proposal of some mechanism that affects ionized helium only. However, further observations are needed, especially including active regions in order to provide a solid support to this suggestion.

This work has been supported by the UK Science and Technology Facilities Council and the University of Strathclyde. The authors thank the SUMER, CDS, and EIS teams for providing new observational sequences and scheduling the observations.

\section{REFERENCES}

Aggarwal, K. M., \& Keenan, F. P. 1999, ApJS, 123, 311

Allard, N., Artru, M.-C., Lanz, T., \& le Dourneuf, M. 1990, A\&AS, 84, 563

Arnaud, M., \& Raymond, J. 1992, ApJ, 398, 394

Arnaud, M., \& Rothenflug, R. 1985, ApJS, 60, 425

Athay, R. G. 1960, ApJ, 131, 705

Badnell, N. R. 1997, JPhB, 30, 1

Ballance, C. P., Badnell, N. R., \& Smith, E. S. 2003, JPhB, 36, 3707

Bhatia, A. K., \& Kastner, S. O. 1988, ApJ, 332, 1063

Bhatia, A. K., \& Landi, E. 2003, ApJ, 585, 587

Bhatia, A. K., \& Landi, E. 2007, ADNDT, 93, 275

Berrington, K. A. 1994, ADNDT, 57, 71

Berrington, K. A., Burke, P. G., Dufton, P. L., \& Kingston, A. E. 1985, ADNDT, 33, 345

Brooks, D. H. 1997, On the Radiating and Dynamic Properties of the Solar Upper Atmosphere, PhD diss., University of Strathclyde

Brooks, D. H., Fischbacher, G. A., Fludra, A., et al. 2000, A\&A, 357, 697

Brooks, D. H., Summers, H. P., Harrison, R. A., Lang, J., \& Lanzafame, A. C. 1999, JASS, 261, 91

Burgess, A., \& Chidichimo, M. C. 1983, MNRAS, 203, 1269

Carlsson, M. 1986, Upps. Astron. Obs. Rep., 33, 1

Cowan, R. D. 1981, The Theory of Atomic Structure and Spectra (Berkeley, CA: Univ. California Press)

Culhane, J. L., Harra, L. K., James, A. M., et al. 2007, SoPh, 243, 19

Curdt, W., Brekke, P., Feldman, U., et al. 2001, A\&A, 375, 591

de Heer, F. J., Hoekstra, R., \& Summers, H. P. 1992, Atomic and Plasma Material Interaction for Fusion, Vol. 3

Dere, K. P., Landi, E., Mason, H. E., Monsignori-Fossi, B. C., \& Young, P. R. 1997, A\&AS, 125, 149

del Zanna, G., Andretta, V., Chamberlin, P. C., Woods, T. N., \& Thompson, W. T. 2010, A\&A, 518, A49

del Zanna, G., Berrington, K. A., \& Mason, H. E. 2004, A\&A, 422, 731

del Zanna, G., \& Mason, H. E. 2005, A\&A, 433, 731

Dufton, P. L., \& Kingston, A. E. 1991, MNRAS, 248, 827

Edlén, B. 1983, PhyS, 28, 48

Edlén, B. 1985, PhyS, 32, 86

Feldman, U., \& Widing, K. G. 2003, SSRv, 107, 665

Feldman, U., \& Widing, K. G. 2007, SSRv, 130, 115

Fischbacher, G. A., Loch, S. D., \& Summers, H. P. 2000, A\&A, 357, 767

Fludra, A., \& Ireland, J. 2008, A\&A, 483, 609

Fludra, A., \& Schmelz, J. T. 1999, A\&A, 348, 286

Fludra, A., \& Warren, H. 2010, A\&A, 523, A47

Froese Fischer, C. 1983, JPhB, 16, 157

Giunta, A. S., Fludra, A., O’Mullane, M. G., \& Summers, H. P. 2012, A\&A, 538, A88

Goldberg, L. 1939, ApJ, 89, 673

Golding, T. P., Carlsson, M., \& Leenaarts, J. 2014, ApJ, 784, 10

Griffin, D. C., Badnell, N. R., \& Pindzola, M. S. 1998, JPhB, 31, 3713

Griffin, D. C., Badnell, N. R., Pindzola, M. S., \& Shaw, J. A. 1999, JPhB, 32,2139

Griffin, D. C., Pindzola, M. S., \& Badnell, N. R. 2000, A\&AS, 142, 317

Harrison, R. A., Sawyer, E. C., Carter, M. K., et al. 1995, SoPh, 162, 233

Harrison, R. A., \& Thompson, A. M. (ed.) 1991, Intensity Integral Inversion

Techniques: A Study in Preparation for the SOHO Mission (Tech. Rep.

RAL-91-092; Chilton: Rutherford Appleton Laboratory)

Hearn, A. G. 1969, MNRAS, 142, 53

Hibbert, A. 1980, JPhB, 13, 1721

Holstein, T. 1947, PhRv, 72, 1212

Jordan, C. 1967, SoPh, 2, 441

Jordan, C. 1975, MNRAS, 170, 429

Jordan, C. 1992, MNRAS, 63, 605

Judge, P. 2008, ApJL, 683, L87 
Judge, P. G., \& Pietarila, A. 2004, ApJ, 606, 1258

Lach, G., \& Pachucki, K. 2001, PhRvA, 64, 042510

Lang, J., Brooks, D. H., Lanzafame, A. C., et al. 2007, A\&A, 463, 339

Lang, J., Kent, B. J., Paustian, W., et al. 2006, ApOpt, 45, 8689

Lang, J., Thompson, W. T., Pike, C. D., Kent, B. J., \& Foley, C. R. 2002, in The Radiometric Calibration of $\mathrm{SOHO}$, ed. A. Pauluhn, M. C. E. Huber, \& R. von Steiger (Noordwijk: ESA), 105, ESA SR-002

Lanzafame, A. C. 1994, A\&A, 287, 972

Lanzafame, A. C., Brooks, D. H., \& Lang, J. 2005, A\&A, 432, 1063

Lanzafame, A. C., Brooks, D. H., Lang, J., et al. 2002, A\&A, 384, 242

Liang, G. Y., Whiteford, A. D., \& Badnell, N. R. 2009, A\&A, 499, 943

Macpherson, K. P., \& Jordan, C. 1999, MNRAS, 308, 510

Malinovsky, M., Dubau, J., \& Sahal-Brechot, S. 1980, ApJ, 235, 665

McLaughlin, B. M., \& Bell, K. L. 1994, ApJS, 94, 825

McWhirter, R. W. P 1965, in Spectral Intensities in Plasma Diagnostic Techniques, ed. R. H. Huddlestone, \& S. L. Leonard (New York: Academic)

McWhirter, R. W. P. 1994, ADNDT, 57, 39

Merkelis, G., Vilkas, M. J., Gaigalas, G., \& Kisielius, R. 1995, PhyS, 51, 233

Meyer, J. P. 1985, ApJS, 57, 173

Moore, C. E. 1993, in Tables of Spectra of Hydrogen, Carbon, Nitrogen and Oxygen Atoms and Ions, ed. J. W. Gallacher (Boca Raton, FL: CRC Press)

Muehlethaler, H. P., \& Nussbaumer, H. 1976, A\&A, 48, 109

Nahar, S. N. 1998, ADNDT, 68, 183
Nussbaumer, H. 1977, A\&A, 58, 291

Paton, I. D. 2005, in On the Neutral Gas Puff as a Tokamak Edge Diagnostic, PhD diss., University of Strathclyde

Phillips, K. J. H., Feldman, U., \& Landi, E. 2008, Ultraviolet and X-ray Spectroscopy of the Solar Atmosphere (Cambridge: Cambridge Univ. Press)

Pietarila, A., \& Judge, P. G. 2004, ApJ, 606, 1239

Ramsbottom, C. A., Bell, K. L., \& Keenan, F. P. 1998, MNRAS, 293, 233

Sampson, D. H., Zhang, H. L., \& Fontes, C. 1990, ADNDT, 44, 209

Sampson, D. H., Zhang, H. L., \& Fontes, C. 1994, ADNDT, 57, 97

Storey, P. J., del Zanna, G., Mason, H. E., \& Zeippen, C. J. 2005, A\&A, 433,717

Storey, P. J., \& Zeippen, C. J. 2010, A\&A, 511, A78

Summers, H. P. 1974, Astrophysics Research Div., RAL, Internal Mem, 367

Summers, H. P. 2001, The ADAS manual, version 2-3, http://adas.phys.strath. ac.uk

Summers, H. P., Dickson, W. J., O’Mullane, M. G., et al. 2006, PPCF, 48, 263 Thompson, A. M. 1990, A\&A, 240, 209

Vernazza, J. E., Avrett, E. H., \& Loeser, R. 1981, ApJS, 45, 635

Wiese, W. L., Fuhr, J. R., \& Deters, T. M. 1996, JPCRD, 7

Wilhelm, K., Curdt, W., Marsch, E., et al. 1995, SoPh, 162, 189

Young, P. R., del Zanna, G., Mason, H. E., et al. 2007, PASJ, 59, S857

Young, P. R., Watanabe, T., Hara, H., \& Mariska, J. T. 2009, A\&A, 495, 587

Zhang, H. L., Sampson, D. H., \& Fontes, C. J. 1990, ADNDT, 44, 31 